\title{
Ehrhart polynomial roots of reflexive polytopes
}

\author{
Gábor Hegedüs \\ Óbuda University \\ Antal Bejczy Center for Intelligent Robotics \\ Budapest H-1032 \\ Hungary \\ hegedus.gabor@nik.uni-obuda.hu
}

\author{
Akihiro Higashitani* \\ Department of Mathematics \\ Graduate School of Science \\ Kyoto Sangyo University \\ Kyoto 603-8555 \\ Japan
}

ahigashi@cc.kyoto-su.ac.jp

\author{
Alexander Kasprzyk ${ }^{\dagger}$ \\ School of Mathematical Sciences \\ University of Nottingham \\ Nottingham NG7 2RD \\ United Kingdom \\ a.m.kasprzyk@nottingham.ac.uk
}

Submitted: Apr 7, 2018; Accepted: Jan 2, 2019; Published: Mar 8, 2019

(C) The authors. Released under the CC BY-ND license (International 4.0).

\begin{abstract}
Recent work has focused on the roots $z \in \mathbb{C}$ of the Ehrhart polynomial of a lattice polytope $P$. The case when $\mathfrak{R e}(z)=-1 / 2$ is of particular interest: these polytopes satisfy Golyshev's "canonical line hypothesis". We characterise such polytopes when $\operatorname{dim}(P) \leqslant 7$. We also consider the "half-strip condition", where all roots $z$ satisfy $-\operatorname{dim}(P) / 2 \leqslant \mathfrak{R e}(z) \leqslant \operatorname{dim}(P) / 2-1$, and show that this holds for any reflexive polytope with $\operatorname{dim}(P) \leqslant 5$. We give an example of a 10-dimensional reflexive polytope which violates the half-strip condition, thus improving on an example by Ohsugi-Shibata in dimension 34.
\end{abstract}

Mathematics Subject Classifications: 52B20, 05A15, 14M25

\section{Introduction}

Let $P \subset \mathbb{Z}^{d} \otimes_{\mathbb{Z}} \mathbb{Q}$ be a convex lattice polytope of dimension $d$. The Ehrhart polynomial [10] $L_{P}$ counts the number of lattice points in successive dilations of $P$, i.e. $L_{P}(m)=\left|m P \cap \mathbb{Z}^{d}\right|$

*Partially supported by a JSPS Fellowship for Young Scientists and by JSPS Grant-in-Aid for Young Scientists (B) $\sharp 17 \mathrm{~K} 14177$.

${ }^{\dagger}$ Supported by EPRSC Fellowship EP/N022513/1. 
for all $m \in \mathbb{Z}_{\geqslant 0}$, and is a polynomial of degree $d$. Stanley [26] showed that the corresponding generating series, the Ehrhart series, can be written as a rational function with numerator a degree $d$ polynomial whose coefficients define the $\delta$-vector (also called the $h^{*}$-vector) of $P$ :

$$
\operatorname{Ehr}_{P}(t)=\frac{\delta_{0}+\delta_{1} t+\ldots+\delta_{d} t^{d}}{(1-t)^{d+1}}=\sum_{m \geqslant 0} L_{P}(m) t^{m} .
$$

Starting with a $\delta$-vector one can easily recover the Ehrhart polynomial:

Lemma 1. Let $P$ be a d-dimensional convex lattice polytope with $\delta$-vector $\left(\delta_{0}, \delta_{1}, \ldots, \delta_{d}\right)$. Then

$$
L_{P}(m)=\sum_{j=0}^{d} \delta_{j}\left(\begin{array}{c}
d+m-j \\
d
\end{array}\right) .
$$

In general, combinatorial interpretations for the coefficients $\delta_{i}$ of the $\delta$-vector are not known, however the work of Ehrhart [11] tells us that:

(i) $\delta_{0}=1$;

(ii) $\delta_{1}=\left|P \cap \mathbb{Z}^{d}\right|-d-1$;

(iii) $\delta_{d}=\left|P^{\circ} \cap \mathbb{Z}^{d}\right|$, where $P^{\circ}=P \backslash \partial P$ denotes the (strict) interior of $P$;

(iv) $\delta_{0}+\ldots+\delta_{d}=d ! \operatorname{vol}(P)$, where $\operatorname{vol}(P)$ denotes the (non-normalised) volume of $P$.

Hibi's Lower Bound Theorem [15] states that if $\left|P^{\circ} \cap \mathbb{Z}^{d}\right|>0$ then $\delta_{1} \leqslant \delta_{i}$ for each $2 \leqslant i \leqslant d-1$. In particular, combined with (ii) we see that the $\delta_{i}$ are positive.

A convex lattice polytope $P$ is called reflexive if the dual (or polar) polyhedron

$$
P^{*}:=\left\{u \in \mathbb{Z}^{d} \otimes_{\mathbb{Z}} \mathbb{Q} \mid\langle u, v\rangle \geqslant-1 \text { for all } v \in P\right\}
$$

is also a lattice polytope. If $P$ is reflexive then $P^{*}$ is also reflexive, and $P^{\circ} \cap \mathbb{Z}^{d}=\{0\}$. Reflexive polytopes are of particular importance in toric geometry: they correspond to Gorenstein toric Fano varieties and are a key combinatorial tool introduced by Batyrev [1] for constructing topologically mirror-symmetric pairs of Calabi-Yau varieties. Reflexive polytopes were characterised by Hibi [14] as being those polytopes (up to lattice translation) with palindromic $\delta$-vectors, i.e. $\delta_{i}=\delta_{d-i}$ for each $0 \leqslant i \leqslant d$. A special class of reflexive polytopes are the smooth Fano polytopes. These are simplicial reflexive polytopes $P$ such that, for each facet $F$, the vertices $\operatorname{vert}(F)$ of $F \mathbb{Z}$-generate the underlying lattice $\mathbb{Z}^{d}$. They correspond to the smooth toric Fano varieties. For a summary of the various equivalences between lattice polytopes and toric Fano varieties, see [19].

Several recent results have concentrated on the roots of the Ehrhart function $L_{P}$, regarded as a polynomial over $\mathbb{C}$. These results are inspired in part by Rodriguez-Villegas' study [25] of Hilbert polynomials all of whose roots $z \in \mathbb{C}$ lie on a line $\mathfrak{R e}(z)=-a / 2$, and the connection when $a=1$ with the Riemann zeta function. Braun [5] has shown 
that the roots of $L_{P}$ lie inside a disc centred at $-1 / 2$ with radius $d(d-1 / 2)$. Beckde Loera-Develin-Pfeifle-Stanley [2] and Braun [6] have also shown that the roots $z$ lie in a strip

$$
-d \leqslant \mathfrak{R e}(z) \leqslant d-1
$$

when $d \leqslant 5$, or for arbitrary $d$ when $\mathfrak{I m}(z)=0$. An example [16] that fails to satisfy the strip condition $(\mathrm{S})$ is known in dimension 15.

When $P$ is a reflexive polytope, Macdonald's Reciprocity Theorem [21] gives that $L_{P}(-m-1)=(-1)^{d} L_{P}(m)$, and so the roots of $L_{P}$ are symmetrically distributed with respect to the line $\mathfrak{R e}(z)=-1 / 2$. Bey-Henk-Wills [3] proved that if

$$
\mathfrak{R e}(z)=-\frac{1}{2}
$$

for all roots $z$ of $L_{P}$ then $P$ is a reflexive polytope (after possible translation by a lattice vector). In fact:

Lemma 2. The lattice polytope $P$ is reflexive (up to lattice translation) if and only if

$$
\sum_{i=1}^{d} z_{i}=-\frac{d}{2}
$$

where $z_{1}, \ldots, z_{d} \in \mathbb{C}$ are the roots of $L_{P}$.

Proof. Write $L_{P}(z)=c_{d} z^{d}+c_{d-1} z^{d-1}+\ldots+c_{0}$. By [11] we have that $c_{d}=\operatorname{vol}(P)$, $c_{d-1}=1 / 2 \operatorname{vol}(\partial P)$, and $c_{0}=1$. Hence:

$$
-\sum_{i=1}^{d} z_{i}=\frac{c_{d-1}}{\operatorname{vol}(P)}=\frac{1}{2} \cdot \frac{\operatorname{vol}(\partial P)}{\operatorname{vol}(P)}
$$

But $P$ is reflexive (up to lattice translation) if and only if $d \operatorname{vol}(P)=\operatorname{vol}(\partial P)[1]$. Hence (1) characterises when a polytope is reflexive.

Golyshev [12] proposed the study of Fano varieties satisfying the so-called canonical line hypothesis; in the context of lattice polytopes this is equivalent to the roots satisfying condition (CL). He conjectured that every $d$-dimensional smooth Fano polytope with $d \leqslant 5$ satisfies the canonical line hypothesis. This was proved in [13], along with an example of a smooth Fano polytope in dimension 6 failing to satisfy (CL). With the above results in mind, we introduce the following terminology:

Definition 3. We say that a lattice polytope $P$ is a $C L$-polytope if the roots $z \in \mathbb{C}$ of the Ehrhart polynomial $L_{P}$ satisfy (CL). We say that $P$ is real if the imaginary part $\mathfrak{I m}(z)=0$ for all roots $z$ of $L_{P}$.

Proposition 4 ([3, Proposition 1.8]). Let $P$ be a d-dimensional CL-polytope. Then $\operatorname{vol}(P) \leqslant 2^{d}$. 
Proof. First we consider the case when $d=2 k$. Let $z_{i} \in \mathbb{C}$ denote the roots of the Ehrhart polynomial $L_{P}$. Then

$$
\prod_{j=1}^{d} z_{j}=\frac{1}{\operatorname{vol}(P)} .
$$

We can assume that $z_{2 j-1}=-1 / 2-\alpha_{j} i$ and $z_{2 j}=-1 / 2+\alpha_{j} i$, where $\alpha_{j} \in \mathbb{R}$, for each $1 \leqslant j \leqslant k$. Hence

$$
\frac{1}{\operatorname{vol}(P)}=\prod_{j=1}^{k} z_{2 j-1} z_{2 j}=\prod_{j=1}^{k}\left(\frac{1}{4}+\alpha_{j}^{2}\right) \geqslant \frac{1}{4^{k}}=\frac{1}{2^{d}} .
$$

The case when $d=2 k+1$ is similar.

In $\S \S 2-4$ we characterise when a reflexive polytope $P$ with $d \leqslant 5$ is a CL-polytope:

Theorem 5 (Canonical line hypothesis). Let $P$ be a d-dimensional reflexive polytope.

$d=2: P$ is a CL-polytope if and only if $\operatorname{vol}(P) \leqslant 4$, or equivalently if and only if $\left|P \cap \mathbb{Z}^{2}\right| \leqslant 9$.

$d=3: P$ is a CL-polytope if and only if $\operatorname{vol}(P) \leqslant 8$, or equivalently if and only if $\left|P \cap \mathbb{Z}^{3}\right| \leqslant 27$.

$d=4: P$ is a CL-polytope if and only if either $\operatorname{vol}(P)=16$ and $\left|P \cap \mathbb{Z}^{4}\right|=81$, or $5 \operatorname{vol}(P)<\left|P \cap \mathbb{Z}^{4}\right|-1,2\left|P \cap \mathbb{Z}^{4}\right| \leqslant 9(\operatorname{vol}(P)+2)$, and $\left(\left|P \cap \mathbb{Z}^{4}\right|-1-4 \operatorname{vol}(P)\right)^{2} \geqslant$ $16 \operatorname{vol}(P)$.

$d=5: P$ is a CL-polytope if and only if either $\operatorname{vol}(P)=32$ and $\left|P \cap \mathbb{Z}^{5}\right|=243$, or $15 \operatorname{vol}(P)<2\left(\left|P \cap \mathbb{Z}^{5}\right|-3\right), 4\left|P \cap \mathbb{Z}^{5}\right| \leqslant 27(\operatorname{vol}(P)+4)$, and $\left(\left|P \cap \mathbb{Z}^{5}\right|-3-\right.$ $6 \operatorname{vol}(P))^{2} \geqslant 72 \operatorname{vol}(P)$.

A characterisation when $d=6$ and 7 is given in $\S 5.1$, Theorems 34 and 35. As a consequence we have:

Corollary 6. Let $P$ be a d-dimensional CL-polytope with $d \leqslant 7$. Then $\left|P \cap \mathbb{Z}^{d}\right| \leqslant 3^{d}$.

We conjecture that Corollary 6 holds for all $d$; if true the result would be sharp, since equality is achieved by the $d$-dimensional cube $\{-1,1\}^{d}$ corresponding to the anticanonical polytope of $\left(\mathbb{P}^{1}\right)^{d}$. In $\S \S 2-4$ we also give characterisations for the real case when $d \leqslant 5$. These characterisations differ from the case of CL-polytopes in Theorem 5 simply by flipping both of the (equivalent) inequalities when $d=2$ or 3 , and by flipping the first inequality when $d=4$ or 5 . In $\S 5.2$, Theorems 37 and 38 , we give characterisations when $d=6$ and 7 ; again these differ from the case for CL-polytopes by flipping certain inequalities.

It was conjectured in [22] that for any reflexive polytope $P$, the roots $z$ of $L_{P}$ satisfy the half-strip condition

$$
-\frac{d}{2} \leqslant \mathfrak{R e}(z) \leqslant \frac{d}{2}-1 .
$$

In $\S 6$ we prove the following: 
Theorem 7. Let $P$ be a d-dimensional reflexive polytope and let $z \in \mathbb{C}$ be a root of $L_{P}$. Inequality (HS) holds when $d \leqslant 5$, or for arbitrary $d$ when $P$ is real.

This result halves the bounds of $(\mathrm{S})$ in the case of reflexive polytopes. OhsugiShibata [23] have found a 34-dimensional reflexive polytope failing to satisfy (HS). In $\S 6.2$ we give a general method for determining whether a palindromic $\delta$-vector with $\delta_{1}=1$ arises from a lattice polytope, and use this in $\S 6.3$ to give a 10 -dimensional example that fails to satisfy (HS). This corresponds to a terminal Gorenstein fake weighted projective space. It seems probable that Theorem 7 also holds when $d=6$ and 7 , and possible that $d=10$ is the smallest dimension in which (HS) fails, although we do not prove this.

\section{A hierarchy of hypotheses}

Golyshev [12] introduced two additional bounds on the roots $z \in \mathbb{C}$ of the Ehrhart polynomial $L_{P}$, which he called the canonical strip hypothesis and the narrowed canonical strip hypothesis. These correspond, respectively, to:

$$
\begin{aligned}
-1 & <\mathfrak{R e}(z)<0 \\
-\frac{d}{d+1} & \leqslant \mathfrak{R e}(z) \leqslant \frac{d}{d+1}-1 .
\end{aligned}
$$

By a slight modification of the proof of Proposition 4 above, we obtain:

Proposition 8. Let $P$ be a d-dimensional real reflexive polytope such that the roots $z \in \mathbb{C}$ of $L_{P}$ satisfy $(\mathrm{CS})$. Then $\operatorname{vol}(P) \geqslant 2^{d}$.

We have a hierarchy of implications

$$
(\mathrm{CL}) \Longrightarrow(\mathrm{NCS}) \Longrightarrow(\mathrm{CS}) \Longrightarrow(\mathrm{HS}) \Longrightarrow(\mathrm{S}) \text {. }
$$

These hypotheses provide meaningful ways of partitioning the space of reflexive polytopes (or, more generally, Fano polytopes) by their $\delta$-vectors. In higher dimensions, where the number of reflexive polytopes is vast, this becomes an essential tool for studying their classification.

\section{Dimensions two and three}

We begin with a general observation. Let $L_{1}:=\{z \in \mathbb{C} \mid \mathfrak{R e}(z)=-1 / 2\}$ and $L_{2}:=\{z \in$ $\mathbb{C} \mid \mathfrak{I m}(z)=0\}$ be lines on the complex plane. By Macdonald's Reciprocity Theorem we see that any reflexive polytope $P$ of dimension $d$ satisfies

$$
L_{P}(-m-1)=(-1)^{d} L_{P}(m) .
$$

In particular the roots of $L_{P}$ are distributed symmetrically with respect to the lines $L_{1}$ and, via complex conjugation, $L_{2}$. When $\operatorname{dim}(P)=2$ or 3 we obtain the following two results (cf. [3, Proposition 1.9(i)]): 
Proposition 9. Let $P$ be a 2 -dimensional reflexive polytope with $\delta$-vector $\left(1, \delta_{1}, 1\right)$. Then either

(i) the roots of $L_{P}$ are $\{-1 / 2 \pm b i\}, b \in \mathbb{R}$, i.e. $P$ is a CL-polytope, or

(ii) the roots of $L_{P}$ are $\{a,-1-a\}, a \in \mathbb{R}$, i.e. $P$ is real.

Furthermore, the following are equivalent:

(ia) $P$ is a CL-polytope; (ib) $\delta_{1} \leqslant 6 ; \quad$ (ic) $\operatorname{vol}(P) \leqslant 4$; and (id) $\left|P \cap \mathbb{Z}^{2}\right| \leqslant 9$.

(iia) $P$ is real; $\quad$ (iib) $\delta_{1} \geqslant 6$; $\quad$ (iic) $\operatorname{vol}(P) \geqslant 4$; and (iid) $\left|P \cap \mathbb{Z}^{2}\right| \geqslant 9$.

In addition, in case (ii) one has that $-1<a<0$, i.e. $P$ satisfies (CS).

Proof. We only give a proof of the last assertion. It follows from Lemma 1 that

$$
L_{P}(z)=\frac{1}{2}\left(\delta_{1}+2\right) z^{2}+\frac{1}{2}\left(\delta_{1}+2\right) z+1 .
$$

The roots of $L_{P}$ are

$$
-\frac{1}{2} \pm \frac{1}{2} \sqrt{\frac{\delta_{1}-6}{\delta_{1}+2}}
$$

Since we have that $\delta_{1} \geqslant 6$ in case (ii), the roots of $L_{P}(z)$ satisfy (CS).

Proposition 10. Let $P$ be a 3-dimensional reflexive polytope with $\delta$-vector $\left(1, \delta_{1}, \delta_{1}, 1\right)$. Then either

(i) the roots of $L_{P}$ are $\{-1 / 2,-1 / 2 \pm b i\}, b \in \mathbb{R}$, i.e. $P$ is a CL-polytope, or

(ii) the roots of $L_{P}$ are $\{-1 / 2, a,-1-a\}, a \in \mathbb{R}$, i.e. $P$ is real.

Furthermore, the following are equivalent:
(ia) $P$ is a CL-polytope;
(ib) $\delta_{1} \leqslant 23 ; \quad$ (ic) $\operatorname{vol}(P) \leqslant 8$; and (id) $\left|P \cap \mathbb{Z}^{3}\right| \leqslant 27$.
(iia) $P$ is real;
(iib) $\delta_{1} \geqslant 23$; (iic) $\operatorname{vol}(P) \geqslant 8$; and (iid) $\left|P \cap \mathbb{Z}^{3}\right| \geqslant 27$.

In addition, in case (ii) one has that $-1<a<0$, i.e. $P$ satisfies (CS).

Proof. It follows from Lemma 1 that

$$
L_{P}(z)=\frac{1}{3}\left(\delta_{1}+1\right) z^{3}+\frac{1}{2}\left(\delta_{1}+1\right) z^{2}+\frac{1}{6}\left(\delta_{1}+13\right) z+1 .
$$

On the other hand, $P$ is a CL-polytope if and only if there exists $b \in \mathbb{R}$ such that

$$
L_{P}(z)=\frac{2+2 \delta_{1}}{3 !}\left(z+\frac{1}{2}\right)\left(z+\frac{1}{2}-b i\right)\left(z+\frac{1}{2}+b i\right) .
$$

By comparing the constant term in the two expressions, we see that $P$ is a CL-polytope if and only if there exists $b \in \mathbb{R}$ such that

$$
4\left(\delta_{1}+1\right) b^{2}+\delta_{1}-23=0 .
$$


Consequently, $P$ is a CL-polytope if and only if $\delta_{1} \leqslant 23$.

A reflexive polytope $P$ is real if and only if there exists $a \in \mathbb{R}$ such that

$$
L_{P}(z)=\frac{2+2 \delta_{1}}{3 !}\left(z+\frac{1}{2}\right)(z-a)(z+1+a) .
$$

Again, comparing the constant term gives $\left(1+\delta_{1}\right) a^{2}+\left(1+\delta_{1}\right) a+6=0$, hence $P$ is real if and only if $\delta_{1} \geqslant 23$. Moreover, since the solutions to this quadratic are given by

$$
-\frac{1}{2} \pm \frac{1}{2} \sqrt{\frac{\delta_{1}-23}{\delta_{1}+1}}
$$

we see that $-1<a<0$ and (CS) is satisfied.

\section{Dimension four}

Let $P$ be a 4 -dimensional reflexive polytope with $\delta$-vector $\left(1, \delta_{1}, \delta_{2}, \delta_{1}, 1\right)$. The roots of $L_{P}$ fall into four possible cases:

(a) The roots of $L_{P}$ are $\left\{-1 / 2 \pm b_{1} i,-1 / 2 \pm b_{2} i\right\}$, where $b_{1}, b_{2} \in \mathbb{R}$. In this case $P$ is a CL-polytope.

(b) The roots of $L_{P}$ are $\left\{-1 / 2 \pm a_{1},-1 / 2 \pm a_{2}\right\}$, where $a_{1}, a_{2} \in \mathbb{R}$. In this case $P$ is real.

(c) The roots of $L_{P}$ are $\{-1 / 2 \pm a,-1 / 2 \pm b i\}$, where $a, b \in \mathbb{R}$.

(d) The roots of $L_{P}$ are $\{-1 / 2+a \pm b i,-1 / 2-a \pm b i\}$, where $a, b \in \mathbb{R} \backslash\{0\}$.

We shall require the following trivial lemma. Although the proof is obvious, we note that one approach that generalises well to higher degree is to employ Descartes' rule of signs.

Lemma 11. Let $A, B, C \in \mathbb{R}$ be real numbers, $A>0$. The solutions of $A z^{2}+B z+C=0$ are non-negative real numbers if and only if either $B=C=0$, or $B<0, C \geqslant 0$, and the discriminant $\Delta \geqslant 0$.

\subsection{Four-dimensional CL-polytopes}

Theorem 12. Let $P$ be a 4-dimensional reflexive polytope with $\delta$-vector $\left(1, \delta_{1}, \delta_{2}, \delta_{1}, 1\right)$. Then $P$ is a CL-polytope (i.e. we are in case (a) above) if and only if either

(i) $\delta_{1}=76$ and $\delta_{2}=230$, or

(ii) $5 \delta_{2}<14 \delta_{1}+86,10 \delta_{1} \leqslant 3 \delta_{2}+70$, and $17\left(\delta_{1}+4 \delta_{2}-15\right)^{2} \leqslant\left(17 \delta_{1}+49\right)^{2}+\left(17 \delta_{2}-94\right)^{2}$.

In particular, if $P$ is a CL-polytope then $\delta_{1} \leqslant 76$ and $\delta_{2} \leqslant 230$. 
Proof. From Lemma 1 we obtain

$$
L_{P}(z)=\left(2+2 \delta_{1}+\delta_{2}\right)\left(\begin{array}{l}
z \\
4
\end{array}\right)+\left(4+4 \delta_{1}+2 \delta_{2}\right)\left(\begin{array}{l}
z \\
3
\end{array}\right)+\left(6+3 \delta_{1}+\delta_{2}\right)\left(\begin{array}{l}
z \\
2
\end{array}\right)+\left(4+\delta_{1}\right)\left(\begin{array}{l}
z \\
1
\end{array}\right)+1 .
$$

Substituting $z=-1 / 2+\beta i$ in (2) and multiplying through by $4 ! / 2$ gives

$$
G(\beta):=\left(1+\delta_{1}+\frac{1}{2} \delta_{2}\right) \beta^{4}-\frac{1}{2}\left(43+7 \delta_{1}-\frac{5}{2} \delta_{2}\right) \beta^{2}+\frac{1}{16}\left(105-15 \delta_{1}+\frac{9}{2} \delta_{2}\right),
$$

and $G(\beta)=0$ if and only if $L_{P}(-1 / 2+\beta i)=0$. Regarding $G$ as a quadratic in $\beta^{2}$ we obtain:

$$
\Delta=17\left(\delta_{1}+\frac{49}{17}\right)^{2}+17\left(\delta_{2}-\frac{94}{17}\right)^{2}-\left(\delta_{1}+4 \delta_{2}-15\right)^{2} .
$$

The two cases follow from Lemma 11. The first two inequalities in case (ii) give $\delta_{1}<76$ and $\delta_{2}<230$; combining these bounds with case (i) we see that $\delta_{1} \leqslant 76$ and $\delta_{2} \leqslant 230$.

The discriminant $\Delta$ gives rise to the parabola $17\left(\delta_{1}+4 \delta_{2}-15\right)^{2}=\left(17 \delta_{1}+49\right)^{2}+\left(17 \delta_{2}-\right.$ $94)^{2}$ with focus at $\left(\delta_{1}, \delta_{2}\right)=(-49 / 17,94 / 17)$. The tangent at the point $(76,230)$ is given by $10 \delta_{1}=3 \delta_{2}+70$. Together these two equations (or, more accurately, the corresponding two inequalities in Theorem 12(ii)) describe three regions in the positive quadrant. The inequality $5 \delta_{2}<14 \delta_{1}+86$ specifies which of these three regions contains the CL-polygons; this is illustrated in Figure 1. There is a choice for this inequality.

Example 13. Case (i) in Theorem 12 can certainly occur: the 4-dimensional cube $\{-1,1\}^{4}$ is a reflexive polytope with $\delta$-vector $(1,76,230,76,1)$. A second example is given in Example 15. It would be an interesting problem to classify all polytopes $P$ with $\delta$-vector equal to the $d$-dimensional cube $\{-1,1\}^{d}$.

Theorem 12 tells us that $\delta_{i} \leqslant \delta_{i}^{\prime}$, for each $0 \leqslant i \leqslant 4$, where $\delta^{\prime}$ is the $\delta$-vector for the 4-dimensional cube $\{-1,1\}^{4}$. In particular we have that $\operatorname{vol}(P) \leqslant 2^{4}$ and $\left|P \cap \mathbb{Z}^{4}\right| \leqslant 3^{4}$.

Example 14. Let $P$ and $Q$ be $d$-dimensional lattice polytopes such that $L_{P}(m)=L_{Q}(2 m)$ for all $m \in \mathbb{Z}_{\geqslant 0}$, and let $\left(\delta_{0}, \delta_{1}, \ldots, \delta_{d}\right)$ be the $\delta$-vector of $Q$. Then

$$
\frac{1}{2}\left(\operatorname{Ehr}_{Q}(t)+\operatorname{Ehr}_{Q}(-t)\right)=\sum_{m \geqslant 0} L_{Q}(2 m) t^{2 m}=\operatorname{Ehr}_{P}\left(t^{2}\right) .
$$

Now

$$
\begin{aligned}
& \frac{1}{2}\left(\operatorname{Ehr}_{Q}(t)+\operatorname{Ehr}_{Q}(-t)\right) \\
& =\frac{(1+t)^{d+1}\left(\delta_{0}+\delta_{1} t+\cdots+\delta_{d} t^{d}\right)+(1-t)^{d+1}\left(\delta_{0}-\delta_{1} t+\cdots+(-1)^{d} \delta_{d} t^{d}\right)}{2\left(1-t^{2}\right)^{d+1}} \\
& =\frac{\sum_{i=0}^{2 d+1} t^{i} \sum_{j=0}^{d}\left(\left(\begin{array}{c}
d+1 \\
i-j
\end{array}\right)+(-1)^{i}\left(\begin{array}{c}
d+1 \\
i-j
\end{array}\right)\right) \delta_{j}}{2\left(1-t^{2}\right)^{d+1}}=\frac{\sum_{i=0}^{d} t^{2 i} \sum_{j=0}^{d}\left(\begin{array}{c}
d+1 \\
2 i-j
\end{array}\right) \delta_{j}}{\left(1-t^{2}\right)^{d+1}},
\end{aligned}
$$


hence the $\delta$-vector of $P$ is given by

$$
\delta_{i}^{P}=\sum_{j=0}^{d}\left(\begin{array}{c}
d+1 \\
2 i-j
\end{array}\right) \delta_{j}, \quad \text { for each } i \in\{0, \ldots, d\} .
$$

Now let $Q=\{0,1\}^{d}$ be the $d$-dimensional cube of unit volume, and let $P=\{-1,1\}^{d}$. It is well-known that the $\delta$-vector of $Q$ can be expressed in terms of the Eulerian numbers, $\delta_{i}=A(d, i)$, hence

$$
\delta_{i}^{P}=\sum_{j=0}^{d}\left(\begin{array}{c}
d+1 \\
2 i-j
\end{array}\right) A(d, j), \quad \text { for each } i \in\{0, \ldots, d\}
$$

Example 15. Let $P=\operatorname{conv}\{(1,0,0,0),(0,1,0,0),(0,0,1,0),(0,0,0,1),(-2,-3,-3,-3)\}$ be the polytope corresponding to weighted projective space $X=\mathbb{P}(1,2,3,3,3)$. The dual polytope $P^{*}$ corresponding to the anticanonical divisor $-K_{X}$ has:

$4 ! \operatorname{vol}\left(P^{*}\right)=\left(-K_{X}\right)^{4}=\frac{(1+2+3+3+3)^{4}}{1 \cdot 2 \cdot 3 \cdot 3 \cdot 3}=384$,

$\left|P^{*} \cap \mathbb{Z}^{4}\right|=h^{0}\left(X_{P},-K_{X}\right)=\#\{$ monomials of weighted degree $1+2+3+3+3=12\}$.

The value of $h^{0}\left(X_{P},-K_{X}\right)$ is given by the coefficient of $t^{12}$ in the Taylor expansion

$$
\frac{1}{(1-t)\left(1-t^{2}\right)\left(1-t^{3}\right)^{3}}=1+t+2 t^{2}+5 t^{3}+\cdots+81 t^{12}+\ldots
$$

We have that $\delta_{1}=76$ and $\delta_{2}=230$, and $P^{*}$ is both a CL-polytope and a real polytope.

Corollary 16. Let $P$ be a 4-dimensional reflexive polytope. Then $P$ is a CL-polytope (i.e. we are in case (a) above) if and only if either

(i) $\operatorname{vol}(P)=16$ and $\left|P \cap \mathbb{Z}^{4}\right|=81$, or

(ii) $5 \operatorname{vol}(P)<\left|P \cap \mathbb{Z}^{4}\right|-1,2\left|P \cap \mathbb{Z}^{4}\right| \leqslant 9(\operatorname{vol}(P)+2)$, and $\left(\left|P \cap \mathbb{Z}^{4}\right|-1-4 \operatorname{vol}(P)\right)^{2} \geqslant$ $16 \operatorname{vol}(P)$.

Proof. Simply make the substitutions $2+2 \delta_{1}+\delta_{2}=4 ! \operatorname{vol}(P)$ and $\delta_{1}=\left|P \cap \mathbb{Z}^{4}\right|-5$ in Theorem 12.

The second and third inequalities in Corollary 16(ii) also appear in the work of BeyHenk-Wills [3, Proposition 1.9(ii)], however they overlook the first inequality. As noted above, this inequality is necessary in order to specify which of the regions we are interested in. In Theorem 17 we will show that flipping this inequality corresponds to selecting the regions containing the real reflexive polytopes. 


\subsection{Four-dimensional real reflexive polytopes}

Theorem 17. Let $P$ be a 4-dimensional reflexive polytope with $\delta$-vector $\left(1, \delta_{1}, \delta_{2}, \delta_{1}, 1\right)$. Then $P$ is a real polytope (i.e. we are in case (b) above) if and only if either

(i) $\delta_{1}=76$ and $\delta_{2}=230$, or

(ii) $5 \delta_{2}>14 \delta_{1}+86,10 \delta_{1} \leqslant 3 \delta_{2}+70$, and $17\left(\delta_{1}+4 \delta_{2}-15\right)^{2} \leqslant\left(17 \delta_{1}+49\right)^{2}+\left(17 \delta_{2}-94\right)^{2}$.

Proof. Let $z=-1 / 2+\alpha, \alpha \in \mathbb{R}$, be a root of $L_{P}$. Substituting this into (2) gives

$$
\frac{\alpha^{4}}{12}\left(1+\delta_{1}+\frac{1}{2} \delta_{2}\right)+\frac{\alpha^{2}}{24}\left(43+7 \delta_{1}-\frac{5}{2} \delta_{2}\right)+\frac{1}{64}\left(35-5 \delta_{1}+\frac{3}{2} \delta_{2}\right)=0
$$

Regarding this as a quadratic in $\alpha^{2}$, the result follows from Lemma 11.

Example 18. Let $Q=\operatorname{conv}\{(-1,2),(-1,-1),(2,-1)\}$ with $L_{Q}(m)=1 / 2(3 m+1)(3 m+$ 2 ). Consider the direct product $P=Q \times Q$. This is a 4-dimensional polytope with Ehrhart polynomial

$$
L_{P}(m)=L_{Q}(m)^{2}=\frac{1}{4}(3 m+1)^{2}(3 m+2)^{2} .
$$

Hence $P$ has $\delta$-vector $(1,95,294,95,1)$ and this gives equality in the third expression in Theorem 17 (ii), i.e. $(95,294)$ is an integer point on the parabola defined by the discriminant $\Delta$.

Example 19. Consider the parabola $17\left(\delta_{1}+4 \delta_{2}-15\right)^{2}=\left(17 \delta_{1}+49\right)^{2}+\left(17 \delta_{2}-94\right)^{2}$. Since we already know an integer solution $\left(\delta_{1}=76, \delta_{2}=230\right)$ we can parameterise the rational points on the curve via:

$$
\delta_{1}=\frac{4\left(19 \gamma^{2}-98 \gamma+124\right)}{(\gamma-4)^{2}}, \quad \delta_{2}=\frac{2\left(223 \gamma^{2}-1280 \gamma+1840\right)}{(\gamma-4)^{2}}, \quad \text { where } \gamma \in \mathbb{Q} .
$$

Interpreting the first of these equations as a quadratic in $\gamma$ and restricting to $\delta_{1} \in \mathbb{Z}$, we see that $\delta_{1}+5$ is a square; this is equivalent to saying that $\left|P \cap \mathbb{Z}^{4}\right|$ is a square. Setting $\delta_{1}+5=\left|P \cap \mathbb{Z}^{4}\right|=N^{2}$ for some $N \in \mathbb{Z}_{\geqslant 1}$ and solving for $\gamma$, we obtain:

$$
\gamma=4-\frac{12}{9 \pm N}
$$

The second equation gives that $\delta_{2}-5$ is a square, and setting $\delta_{2}-5=M^{2}$ for some $M \in \mathbb{Z}_{\geqslant 1}$ we obtain:

$$
\gamma=4-\frac{24}{21 \pm M}
$$

Equating these two expressions for $\gamma$, and remembering that $\left|P \cap \mathbb{Z}^{4}\right| \geqslant 6$, we find that

$$
\left(\delta_{1}, \delta_{2}\right)=\left(N^{2}-5,(2 N \pm 3)^{2}+5\right), \quad \text { for } N \geqslant 3 .
$$

By consulting the Kreuzer-Skarke classification [20] we see that the cases with $\delta_{2}=(2 \mathrm{~N}+$ $3)^{2}+5$ never occur (this corresponds to the upper branch of the parabola); the reflexive polytopes lie on the bottom branch with $\delta_{2}=(2 N-3)^{2}+5$, for each $N \in\{3, \ldots, 13\}$. When $N>13$ there are no matching $\delta$-vectors. The occurring $\delta$-vectors are recorded in Table 1. 


\begin{tabular}{cccccccccccc}
\hline$N$ & 3 & 4 & 5 & 6 & 7 & 8 & 9 & 10 & 11 & 12 & 13 \\
\hline$\delta_{1}$ & 4 & 11 & 20 & 31 & 44 & 59 & 76 & 95 & 116 & 139 & 164 \\
$\delta_{2}$ & 14 & 30 & 54 & 86 & 126 & 174 & 230 & 294 & 366 & 446 & 534 \\
\hline
\end{tabular}

Table 1: The $\delta$-vectors of the 4-dimensional reflexive polytopes lying on the parabola $17\left(\delta_{1}+4 \delta_{2}-15\right)^{2}=\left(17 \delta_{1}+49\right)^{2}+\left(17 \delta_{2}-94\right)^{2}$. Those with $3 \leqslant N \leqslant 9$ correspond to CL-polytopes; those with $9 \leqslant N \leqslant 13$ correspond to real polytopes.

Corollary 20. Let $P$ be a 4-dimensional reflexive polytope. Then $P$ is a real polytope (i.e. we are in case (b) above) if and only if either

(i) $\operatorname{vol}(P)=16$ and $\left|P \cap \mathbb{Z}^{4}\right|=81$, or

(ii) $5 \operatorname{vol}(P)>\left|P \cap \mathbb{Z}^{4}\right|-1,2\left|P \cap \mathbb{Z}^{4}\right| \leqslant 9(\operatorname{vol}(P)+2)$, and $\left(\left|P \cap \mathbb{Z}^{4}\right|-1-4 \operatorname{vol}(P)\right)^{2} \geqslant$ $16 \operatorname{vol}(P)$.

Proposition 21. Let $P$ be a 4-dimensional real reflexive polytope. Then $\operatorname{vol}(P) \geqslant 3$.

Proof. By Theorem 17, $P$ is real if and only if $\delta_{1}, \delta_{2}$ satisfy either conditions (i) or (ii). In case (i), since $\operatorname{vol}(P)=\left(2+2 \delta_{1}+\delta_{2}\right) / 24$, there is nothing to prove. In case (ii), by the inequality $17\left(\delta_{1}+4 \delta_{2}-15\right)^{2} \leqslant\left(17 \delta_{1}+49\right)^{2}+\left(17 \delta_{2}-94\right)^{2}$ we have $16 \delta_{1}^{2}-8\left(\delta_{2}-16\right) \delta_{1}+$ $\delta_{2}^{2}-68 \delta_{2}+436 \geqslant 0$, that is:

$$
\delta_{1} \geqslant \frac{\left(\delta_{2}-16\right)+6 \sqrt{\delta_{2}-5}}{4}, \quad \text { or } \quad 1 \leqslant \delta_{1} \leqslant \frac{\left(\delta_{2}-16\right)-6 \sqrt{\delta_{2}-5}}{4} .
$$

Moreover, by the inequality $5 \delta_{2}>14 \delta_{1}+86$, we have that $\delta_{1}<\left(5 \delta_{2}-86\right) / 14$.

When the first condition in (4) is satisfied, and recalling that $\delta_{1} \leqslant \delta_{2}$, we see that

$$
\frac{\left(\delta_{2}-16\right)+6 \sqrt{\delta_{2}-5}}{4} \leqslant \delta_{1}<\frac{5 \delta_{2}-86}{14}
$$

and obtain $\delta_{2}>230$. When the second condition in (4) is satisfied, since $\left(\delta_{2}-16-\right.$ $\left.6 \sqrt{\delta_{2}-5}\right) / 4 \geqslant 1$, we obtain $\delta_{2} \geqslant\lceil 38+12 \sqrt{6}\rceil=68$. Hence $\left(2+2 \delta_{1}+\delta_{2}\right) / 24 \geqslant(2+2+$ $68) / 24=3$, as required.

By the proof of Proposition 21, $P$ is a 4-dimensional real reflexive polytope with $\operatorname{vol}(P)=3$ if and only if $\delta_{P}=(1,1,68,1,1)$. Using the method described in $\S 6.2$ we can show that no such $P$ exists.

The 4-dimensional reflexive polytopes were classified by Kreuzer-Skarke [20]: there are 473800776 cases. Corollary 20 makes extracting the real reflexive polytopes a simple matter, and we can recover their $\delta$-vectors. We find that the region to the left of the parabola (and closest to the $\delta_{2}$-axis) in Figure 1 is empty: all the $\delta$-vectors lie in the narrow region between the parabola and the tangent. A plot of all of the $\delta$-vectors suggests very strongly that there is an additional inequality awaiting discovery. Furthermore: 


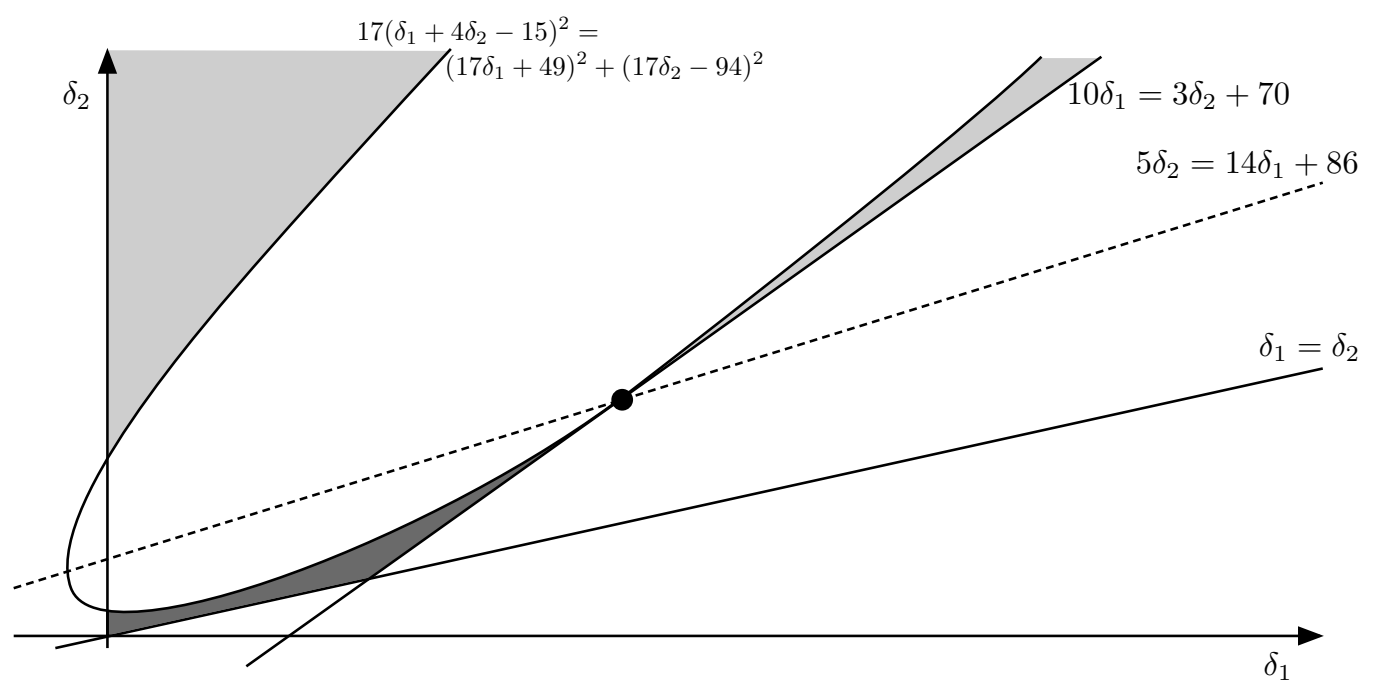

Figure 1: An illustration of the regions containing the pairs $\left(\delta_{1}, \delta_{2}\right)$ for the fourdimensional CL-polytopes (the darker, bounded region closest to the origin) and the real reflexive polytopes (the lighter, unbounded regions on either side of the parabola). The dot near the centre represents the value $(76,230)$, corresponding to the Ehrhart $\delta$-vector of the 4-cube, and gives polytopes which are simultaneously both CL-polytopes and real. The line $10 \delta_{1}=3 \delta_{2}+70$ is tangent to the parabola at this point.

Proposition 22. Let $P$ be a 4-dimensional real reflexive polytope. Then the roots of $L_{P}$ satisfy (CS).

As a consequence, Proposition 8 tells us that $\operatorname{vol}(P) \geqslant 2^{4}$. Unfortunately we do not have a theoretical explanation for Proposition 22.

\subsection{Remaining cases}

Proposition 23. Let $P$ be a 4-dimensional reflexive polytope, and suppose that there are two roots of $L_{P}$ which are real, and that there are two roots with real part $-1 / 2$ (i.e. we are in case (c) above). Then $\operatorname{vol}(P) \geqslant 4 / 3$.

Proof. By considering the left-hand side of (3) we see that we are in case (c) if and only if $70-10 \delta_{1}+3 \delta_{2} \leqslant 0$. Since $\delta_{1} \leqslant \delta_{2}$, we have that $7\left(10-\delta_{1}\right) \leqslant 70-10 \delta_{1}+3 \delta_{2} \leqslant 0$. Thus $\delta_{1} \geqslant 10$. Therefore $\operatorname{vol}(P)=\left(2+2 \delta_{1}+\delta_{2}\right) / 24 \geqslant\left(2+3 \delta_{1}\right) / 24 \geqslant 4 / 3$, as required.

Proposition 24. Let $P$ be a 4-dimensional reflexive polytope, and suppose that there exists a root $-1 / 2+a+b i \in \mathbb{C}$ of $L_{P}$, where $a \neq 0$ and $b \neq 0$ (i.e. we are in case (d) above). Then $|a|<3 / 2$ and the roots of $L_{P}$ satisfy (HS).

Proof. Consider $\alpha=a+b i \in \mathbb{C}$ satisfying (3). We will show that $\alpha$ satisfies $-3 / 2<$ $\mathfrak{R e}(\alpha)<3 / 2$. Let

$$
F(z)=\left(2+2 \delta_{1}+\delta_{2}\right) z^{2}+\left(43+7 \delta_{1}-\frac{5}{2} \delta_{2}\right) z+\frac{105}{8}-\frac{15}{8} \delta_{1}+\frac{9}{16} \delta_{2}
$$


and consider the roots of $F$. By our hypothesis we have that $\Delta(F)<0$. Let $\beta$ and $\gamma$ be the roots of $F$, and write $\beta=r e^{\theta i}$ with $r>0$ and $0<\theta<\pi$, so that $\gamma=\bar{\beta}=r e^{-\theta i}$. Thus the roots $\alpha$ are given by

$$
\sqrt{r} e^{\frac{\theta}{2} i}, \quad \sqrt{r} e^{\left(\pi-\frac{\theta}{2}\right) i}, \quad \sqrt{r} e^{-\frac{\theta}{2} i}, \quad \text { and } \quad \sqrt{r} e^{-\left(\pi-\frac{\theta}{2}\right) i} .
$$

Hence it is sufficient to show that

$$
0<\mathfrak{R e}\left(\sqrt{r} e^{\frac{\theta}{2} i}\right)=\sqrt{r} \cos \frac{\theta}{2}=\sqrt{\frac{r+r \cos \theta}{2}} \leqslant \frac{3}{2} .
$$

Since $F(z)=\left(2+2 \delta_{1}+\delta_{2}\right)(z-\beta)(z-\gamma)$, we have

$$
r=\sqrt{\beta \gamma}=\frac{1}{4} \sqrt{\frac{210-30 \delta_{1}+9 \delta_{2}}{2+2 \delta_{1}+\delta_{2}}} .
$$

Moreover, one has

$$
\Delta(F)=4\left(16 \delta_{1}^{2}-8\left(\delta_{2}-16\right) \delta_{1}+\delta_{2}^{2}-68 \delta_{2}+436\right) .
$$

Let $h\left(\delta_{1}\right):=\Delta(F) / 4$ be regarded as a quadratic in $\delta_{1}$. The range of $\delta_{1}$ such that $h\left(\delta_{1}\right)<0$ is given by:

$$
\frac{\delta_{2}-16-6 \sqrt{\delta_{2}-5}}{4}<\delta_{1}<\frac{\delta_{2}-16+6 \sqrt{\delta_{2}-5}}{4} .
$$

Since $\delta_{1} \geqslant 1$, we conclude that $\left(\delta_{2}-16+6 \sqrt{\delta_{2}-5}\right) / 4>1$. Hence $\delta_{2}>38-12 \sqrt{6}$, and the condition that $\Delta(F)<0$ is equivalent to:

$$
\begin{aligned}
& \delta_{2}>38-12 \sqrt{6}, \quad \text { and } \\
& \begin{cases}1 \leqslant \delta_{1}<\frac{\delta_{2}-16+6 \sqrt{\delta_{2}-5}}{4}, & \text { when } \delta_{2} \leqslant 38+12 \sqrt{6} ; \\
\frac{\delta_{2}-16-6 \sqrt{\delta_{2}-5}}{4}<\delta_{1}<\frac{\delta_{2}-16+6 \sqrt{\delta_{2}-5}}{4}, & \text { when } \delta_{2}>38+12 \sqrt{6} .\end{cases}
\end{aligned}
$$

When $\delta_{1}$ and $\delta_{2}$ satisfy the first condition of (5), we have:

$$
\begin{aligned}
\frac{210-30 \delta_{1}+9 \delta_{2}}{2+2 \delta_{1}+\delta_{2}}=-15+\frac{24\left(\delta_{2}+10\right)}{2+2 \delta_{1}+\delta_{2}} & \leqslant-15+\frac{24\left(\delta_{2}+10\right)}{\delta_{2}+4} \\
& <-15+\frac{24(38-12 \sqrt{6}+10)}{38-12 \sqrt{6}+4}<81 .
\end{aligned}
$$

When the second condition of (5) is satisfied, we have:

$$
\begin{aligned}
\frac{210-30 \delta_{1}+9 \delta_{2}}{2+2 \delta_{1}+\delta_{2}} & =-15+\frac{24\left(\delta_{2}+10\right)}{2+2 \delta_{1}+\delta_{2}} \\
& <-15+\frac{24\left(\delta_{2}+10\right)}{\frac{\delta_{2}-16-6 \sqrt{\delta_{2}-5}}{2}+\delta_{2}+2}=-15+\frac{16\left(\delta_{2}+10\right)}{\delta_{2}-4-2 \sqrt{\delta_{2}-5}}\left(=: H\left(\delta_{2}\right)\right) \\
& <-15+\frac{16(38+12 \sqrt{6}+10)}{38+12 \sqrt{6}-4-2 \sqrt{38+12 \sqrt{6}-5}} \\
& <81 .
\end{aligned}
$$


Here we make use of the fact that $d H\left(\delta_{2}\right) / d \delta_{2}<0$ if $\delta_{2}>38+12 \sqrt{6}$. We conclude that

$$
\sqrt{\frac{r+r \cos \theta}{2}} \leqslant \sqrt{r}=\frac{1}{2}\left(\frac{210-30 \delta_{1}+9 \delta_{2}}{2+2 \delta_{1}+\delta_{2}}\right)^{\frac{1}{4}}<\frac{3}{2}
$$

as required.

\section{Dimension five}

Let $P$ be a 5 -dimensional reflexive polytope with $\delta$-vector $\left(1, \delta_{1}, \delta_{2}, \delta_{2}, \delta_{1}, 1\right)$. One of the roots of $L_{P}$ is $-1 / 2$. The remaining roots fall into the four possible cases (a)-(d) described at the beginning of $\S 3$.

\subsection{Five-dimensional CL-polytopes}

Theorem 25. Let $P$ be a 5-dimensional reflexive polytope with $\delta$-vector $\left(1, \delta_{1}, \delta_{2}, \delta_{2}, \delta_{1}, 1\right)$. Then $P$ is a CL-polytope if and only if either

(i) $\delta_{1}=237$ and $\delta_{2}=1682$, or

(ii) $\delta_{2}<7 \delta_{1}+23,71 \delta_{1} \leqslant 9 \delta_{2}+1689$, and $41\left(\delta_{1}+9 \delta_{2}-9\right)^{2} \leqslant 2\left(41 \delta_{1}+96\right)^{2}+2\left(41 \delta_{2}-85\right)^{2}$.

In particular, if $P$ is a CL-polytope then $\delta_{1} \leqslant 237$ and $\delta_{2} \leqslant 1682$.

Proof. From Lemma 1 we have

$$
L_{P}(z)=\left(\begin{array}{c}
z+5 \\
5
\end{array}\right)+\left(\begin{array}{l}
z \\
5
\end{array}\right)+\delta_{1}\left(\left(\begin{array}{c}
z+4 \\
5
\end{array}\right)+\left(\begin{array}{c}
z+1 \\
5
\end{array}\right)\right)+\delta_{2}\left(\left(\begin{array}{c}
z+3 \\
5
\end{array}\right)+\left(\begin{array}{c}
z+2 \\
5
\end{array}\right)\right) .
$$

Since $-1 / 2$ is a root, we know that $L_{P}$ is divisible by $z+1 / 2$. Set $f(z):=5 ! L_{P}(z) /(z+1 / 2)$. Substituting $z=-1 / 2+\beta i$ in $f$ and multiplying through by 5 ! gives

$$
G(\beta):=2\left(1+\delta_{1}+\delta_{2}\right) \beta^{4}-5\left(23+7 \delta_{1}-\delta_{2}\right) \beta^{2}+\frac{1}{8}\left(1689-71 \delta_{1}+9 \delta_{2}\right) .
$$

This is a quadratic in $\beta^{2}$, and $G(\beta)=0$ if and only if $L_{P}(-1 / 2+\beta i)=0$. The two cases follow from Lemma 11. By combining the first two inequalities in case (ii) we see that $\delta_{1}<237$ and $\delta_{2}<1682$; including case (i) we have that $\delta_{1} \leqslant 237$ and $\delta_{2} \leqslant 1682$.

Once more we see that the inequalities in Theorem 25(ii) are determined by the discriminant $\Delta$ of $G$ : the parabola $41\left(\delta_{1}+9 \delta_{2}-9\right)^{2}=2\left(41 \delta_{1}+96\right)^{2}+2\left(41 \delta_{2}-85\right)^{2}$. The tangent at the point $\left(\delta_{1}, \delta_{2}\right)=(237,1682)$ is given by $71 \delta_{1}=9 \delta_{2}+1689$, and together these two equations (or, more accurately, the corresponding two inequalities) cut out three regions in the positive quadrant. The inequality $\delta_{2}<7 \delta_{1}+23$ distinguishes which of these regions contains the $\delta$-vectors for the CL-polygons and, as we shall see in Theorem 27 below, which contains the real reflexive polygons. The situation is essentially the same as the case in 4-dimensions illustrated in Figure 1. As in the 4-dimensional case, we expect there to be a "missing inequality" that excludes the top-left-most region from consideration. 
Corollary 26. Let $P$ be a 5-dimensional reflexive polytope. Then $P$ is a CL-polytope if and only if either

(i) $\operatorname{vol}(P)=32$ and $\left|P \cap \mathbb{Z}^{5}\right|=243$, or

(ii) $15 \operatorname{vol}(P)<2\left(\left|P \cap \mathbb{Z}^{5}\right|-3\right), 4\left|P \cap \mathbb{Z}^{5}\right| \leqslant 27(\operatorname{vol}(P)+4)$, and $\left(\left|P \cap \mathbb{Z}^{5}\right|-3\right.$ $6 \operatorname{vol}(P))^{2} \geqslant 72 \operatorname{vol}(P)$.

\subsection{Five-dimensional real reflexive polytopes}

Theorem 27. Let $P$ be a 5-dimensional reflexive polytope with $\delta$-vector $\left(1, \delta_{1}, \delta_{2}, \delta_{2}, \delta_{1}, 1\right)$. Then $P$ is real if and only if either

(i) $\delta_{1}=237$ and $\delta_{2}=1632$, or

(ii) $\delta_{2}>7 \delta_{1}+23,71 \delta_{1} \leqslant 9 \delta_{2}+1689$, and $41\left(\delta_{1}+9 \delta_{2}-9\right)^{2} \leqslant 2\left(41 \delta_{1}+96\right)^{2}+2\left(41 \delta_{2}-85\right)^{2}$.

Proof. Substituting $z=-1 / 2+\alpha$, where $\alpha \in \mathbb{R}$, into $f(z)$ in the proof of Theorem 25 gives

$$
2\left(1+\delta_{1}+\delta_{2}\right) \alpha^{4}+5\left(23+7 \delta_{1}-\delta_{2}\right) \alpha^{2}+\frac{1}{8}\left(1689-71 \delta_{1}+9 \delta_{2}\right)=0 .
$$

This is a quadratic in $\alpha^{2}$ and the result follows from Lemma 11.

Corollary 28. Let $P$ be a 5-dimensional reflexive polytope. Then $P$ is real if and only if either

(i) $\operatorname{vol}(P)=32$ and $\left|P \cap \mathbb{Z}^{5}\right|=243$, or

(ii) $15 \operatorname{vol}(P)>2\left(\left|P \cap \mathbb{Z}^{5}\right|-3\right), 4\left|P \cap \mathbb{Z}^{5}\right| \leqslant 27(\operatorname{vol}(P)+4)$, and $\left(\left|P \cap \mathbb{Z}^{5}\right|-3\right.$ $6 \operatorname{vol}(P))^{2} \geqslant 72 \operatorname{vol}(P)$.

Proposition 29. Let $P$ be a 5-dimensional real reflexive polytope. Then $\operatorname{vol}(P) \geqslant 16 / 5$.

Proof. By Theorem 27, $P$ is real if and only if $\delta_{1}$ and $\delta_{2}$ satisfy either condition (i), or condition (ii). In case (i), since $\operatorname{vol}(P)=2\left(1+\delta_{1}+\delta_{2}\right) / 120$, we are done. In case (ii), by the third inequality $41\left(\delta_{1}+9 \delta_{2}-9\right)^{2} \leqslant 2\left(41 \delta_{1}+96\right)^{2}+2\left(41 \delta_{2}-85\right)^{2}$, we have

$$
\delta_{1} \geqslant \frac{3 \delta_{2}-67+20 \sqrt{3 \delta_{2}-5}}{27}, \quad \text { or } \quad 1 \leqslant \delta_{1} \leqslant \frac{3 \delta_{2}-67-20 \sqrt{3 \delta_{2}-5}}{27} .
$$

Moreover, by inequality $\delta_{2}>7 \delta_{1}+23$, we have that $\delta_{1}<\left(\delta_{2}-23\right) / 7$.

When the first condition in (7) is satisfied, using $\delta_{1} \leqslant \delta_{2}$ we see that

$$
\frac{3 \delta_{2}-67+20 \sqrt{3 \delta_{2}-5}}{27} \leqslant \delta_{1}<\frac{\delta_{2}-23}{7} .
$$

Hence we obtain $\delta_{2}>1682$, and so we are done. When the second condition in (7) is satisfied, using the fact that $\left(3 \delta_{2}-67-20 \sqrt{3 \delta_{2}-5}\right) / 27 \geqslant 1$, we obtain $\delta_{2} \geqslant\lceil 98+$ $20 \sqrt{21}\rceil=190$. Hence $2\left(1+\delta_{1}+\delta_{2}\right) / 120 \geqslant(1+1+190) / 60=16 / 5$, as required.

By the proof of Proposition 29, $P$ is a 5-dimensional real reflexive polytope with $\operatorname{vol}(P)=16 / 5$ if and only if $\delta=(1,1,190,190,1,1)$. One can show that no such $P$ exists by the method of $\S 6.2$ below. 


\subsection{Remaining cases}

Proposition 30. Let $P$ be a 5-dimensional polytope, and suppose that there are three roots of $L_{P}$ which are real, and that there are two imaginary roots with real part $-1 / 2$. Then $\operatorname{vol}(P) \geqslant 19 / 20$.

Proof. By considering the left-hand side of (6), we see that there are two real roots and two roots with real part $-1 / 2$ if and only if $1689-71 \delta_{1}+9 \delta_{2} \leqslant 0$. Since $\delta_{1} \leqslant \delta_{2}$, we have that $0 \geqslant 1689-71 \delta_{1}+9 \delta_{2} \geqslant 1689-62 \delta_{1}$. Thus $\delta_{1} \geqslant\lceil 1689 / 62\rceil=28$. Therefore $\operatorname{vol}(P)=2\left(1+\delta_{1}+\delta_{2}\right) / 120 \geqslant\left(1+2 \delta_{1}\right) / 60 \geqslant 19 / 20$.

Proposition 31. Let $P$ be a 5-dimensional reflexive polytope, and suppose that there exists a root $-1 / 2+a+b i \in \mathbb{C}$ of $L_{P}$, where $a \neq 0$ and $b \neq 0$. Then $|a|<3 / 2$ and the roots of $L_{P}$ satisfy (HS).

Proof. A proof can be done in the similar way to that of Proposition 24. We consider $\alpha$ satisfying equation (6); we need to prove that $\alpha$ satisfies $-3 / 2<\mathfrak{R e}(\alpha)<3 / 2$. Set

$$
F(z):=2\left(1+\delta_{1}+\delta_{2}\right) z^{2}+5\left(23+7 \delta_{1}-\delta_{2}\right) z+\frac{1689}{8}-\frac{71}{8} \delta_{1}+\frac{9}{8} \delta_{2},
$$

and consider the roots of $F$. By the hypothesis we have that $\Delta(F)<0$. We shall show that

$$
\sqrt{\frac{r+r \cos \theta}{2}}<\frac{3}{2}
$$

where $\beta=r e^{\theta i}$ is a root of $F, r>0,0<\theta<\pi$. Notice that

$$
r=\sqrt{\beta \bar{\beta}}=\frac{1}{4} \sqrt{\frac{1689-71 \delta_{1}+9 \delta_{2}}{1+\delta_{1}+\delta_{2}}}, \quad \text { and } \quad r \cos \theta=\frac{\beta+\bar{\beta}}{2}=-\frac{1}{4} \cdot \frac{5\left(23+7 \delta_{1}-\delta_{2}\right)}{1+\delta_{1}+\delta_{2}} .
$$

Moreover, one has that

$$
\Delta(F)=\frac{16}{41}\left(2\left(41 \delta_{1}+96\right)^{2}+2\left(41 \delta_{2}-85\right)^{2}-41\left(\delta_{1}+9 \delta_{2}-9\right)^{2}\right) .
$$

Set $h\left(\delta_{1}\right):=\Delta(F) / 16$ to be a polynomial in $\delta_{1}$. We require that $h\left(\delta_{1}\right)<0$. The range of values for $\delta_{1}$ such that $h\left(\delta_{1}\right)<0$ is:

$$
\frac{3 \delta_{2}-67-20 \sqrt{3 \delta_{2}-5}}{27}<\delta_{1}<\frac{3 \delta_{2}-67+20 \sqrt{3 \delta_{2}-5}}{27} .
$$

Since $\delta_{1} \geqslant 1$, we have that $\left(3 \delta_{2}-67+20 \sqrt{3 \delta_{2}-5}\right) / 27>1$. Thus $\delta_{2}>98-20 \sqrt{21}$. Hence the condition $\Delta(F)<0$ is equivalent to:

$$
\begin{aligned}
& \delta_{2}>98-20 \sqrt{21}, \quad \text { and } \\
& \begin{cases}1 \leqslant \delta_{1}<\frac{3 \delta_{2}-67+20 \sqrt{3 \delta_{2}-5}}{27}, & \text { when } \delta_{2} \leqslant 98+20 \sqrt{21} \\
\frac{3 \delta_{2}-67-20 \sqrt{3 \delta_{2}-5}}{27}<\delta_{1}<\frac{3 \delta_{2}-67+20 \sqrt{3 \delta_{2}-5}}{27}, & \text { when } \delta_{2}>98+20 \sqrt{21}\end{cases}
\end{aligned}
$$


When the first condition of (8) is satisfied, we have:

$$
\begin{aligned}
\sqrt{\frac{1689-71 \delta_{1}+9 \delta_{2}}{1+\delta_{1}+\delta_{2}}-} & \frac{5\left(23+7 \delta_{1}-\delta_{2}\right)}{1+\delta_{1}+\delta_{2}} \\
& =\sqrt{-71+\frac{80\left(\delta_{2}+22\right)}{1+\delta_{1}+\delta_{2}}}+\frac{40\left(\delta_{2}-2\right)}{1+\delta_{1}+\delta_{2}}-35 \\
& \leqslant \sqrt{-71+\frac{80\left(\delta_{2}+22\right)}{\delta_{2}+2}}+\frac{40\left(\delta_{2}-2\right)}{\delta_{2}+2}-35 \quad\left(=: H_{1}\left(\delta_{2}\right)\right) \\
& \leqslant \sqrt{-71+\frac{80(98+22)}{98+2}}+\frac{40(98-2)}{98+2}-35 \\
& \left(\text { since } \frac{d H_{1}\left(\delta_{2}\right)}{d \delta_{2}}>0 \text { if } \delta_{2}<98 \text { and } \frac{d H_{1}\left(\delta_{2}\right)}{d \delta_{2}}<0 \text { if } \delta_{2}>98\right) \\
& <18
\end{aligned}
$$

We obtain:

$$
\sqrt{\frac{r+r \cos \theta}{2}}<\sqrt{\frac{18}{4} \cdot \frac{1}{2}}=\frac{3}{2}
$$

When the second condition of (8) is satisfied, we have:

$$
\begin{aligned}
\frac{1689-71 \delta_{1}+9 \delta_{2}}{1+\delta_{1}+\delta_{2}} & =-71+\frac{80\left(\delta_{2}+22\right)}{1+\delta_{1}+\delta_{2}} \\
& <-71+\frac{80\left(\delta_{2}+22\right)}{\frac{3 \delta_{2}-67-20 \sqrt{3 \delta_{2}-5}}{27}+\delta_{2}+2} \\
& =-71+\frac{2160\left(\delta_{2}+22\right)}{30 \delta_{2}-13-20 \sqrt{3 \delta_{2}-5}} \quad\left(=: H_{2}\left(\delta_{2}\right)\right) \\
& <-71+\frac{2160(98+20 \sqrt{21}+22)}{30(98+20 \sqrt{21})-18-20 \sqrt{3(98+20 \sqrt{21})}} \\
& \left(\text { since } \frac{d H_{2}\left(\delta_{2}\right)}{d \delta_{2}}<0 \text { if } \delta_{2} \geqslant 98+20 \sqrt{21}\right) \\
& <81 .
\end{aligned}
$$

We conclude that $\sqrt{\frac{r+r \cos \theta}{2}}<\frac{3}{2}$, as required.

\section{Dimensions six and seven}

If $P$ is a $(2 k+1)$-dimensional reflexive polytope then $-1 / 2$ is a root of $L_{P}$. We shall consider both 6 - and 7-dimensional reflexive polytopes together. We shall require the following two elementary lemmas: Lemma 32 is an application of Descartes' rule of signs; Lemma 33 follows from Rouche's Theorem. 
Lemma 32. Let $A, B, C, D \in \mathbb{R}$ be real numbers, $A>0$. All solutions of the equation $A z^{3}+B z^{2}+C z+D=0$ are positive real numbers if and only if $B<0, C>0, D \leqslant 0$, and the discriminant $\Delta \geqslant 0$.

Lemma 33. Let $A, B, C, D \in \mathbb{R}$ be real numbers, $A>0$. All solutions of the equation $A z^{3}+B z^{2}+C z+D=0$ are non-negative real numbers if and only if one of the following conditions is satisfied:

(i) $B=C=D=0$;

(ii) $B<0$ and $C=D=0$; or

(iii) $\Delta \geqslant 0, B<0, C>0$, and $D<0$.

We will also make use of the following two polynomials:

$$
\begin{aligned}
f_{6}(z):=(2+ & \left.2 \delta_{1}+2 \delta_{2}+\delta_{3}\right) z^{3}-\frac{5}{4}\left(202+82 \delta_{1}+10 \delta_{2}-7 \delta_{3}\right) z^{2} \\
& +\frac{1}{16}\left(24278+1478 \delta_{1}-682 \delta_{2}+259 \delta_{3}\right) z-\frac{45}{64}\left(462-42 \delta_{1}+14 \delta_{2}-5 \delta_{3}\right) \\
f_{7}(z):=(1+ & \left.\delta_{1}+\delta_{2}+\delta_{3}\right) z^{3}-\frac{7}{4}\left(139+67 \delta_{1}+19 \delta_{2}-5 \delta_{3}\right) z^{2} \\
& +\frac{7}{16}\left(8197+1237 \delta_{1}-203 \delta_{2}+37 \delta_{3}\right) z-\frac{3}{64}\left(88069-3043 \delta_{1}+429 \delta_{2}-75 \delta_{3}\right)
\end{aligned}
$$

\subsection{Six- and seven-dimensional CL-polytopes}

Theorem 34. Let $P$ be a 6-dimensional reflexive polytope with $\delta$-vector

$$
\left(1, \delta_{1}, \delta_{2}, \delta_{3}, \delta_{2}, \delta_{1}, 1\right)
$$

Then $P$ is a CL-polytope if and only if one of the following holds:

(i) $\delta_{1}=722, \delta_{2}=10543$, and $\delta_{3}=23548$;

(ii) $994 \delta_{1}=27 \delta_{3}+81872,497 \delta_{2}=218 \delta_{3}+106407$, and $\delta_{3}<23548$; or

(iii) $202+82 \delta_{1}+10 \delta_{2}>7 \delta_{3}, 24278+1478 \delta_{1}+259 \delta_{3}>682 \delta_{2}, 462+14 \delta_{2}>42 \delta_{1}+5 \delta_{3}$, and $\Delta\left(f_{6}\right) \geqslant 0$.

In particular, if $P$ is a CL-polytope then $\delta_{1} \leqslant 722, \delta_{2} \leqslant 10543$, and $\delta_{3} \leqslant 23548$.

Proof. From Lemma 1 we obtain

$$
\begin{aligned}
L_{P}(z)=\left(\begin{array}{c}
z+6 \\
6
\end{array}\right)+\left(\begin{array}{l}
z \\
6
\end{array}\right)+ & \delta_{1}\left(\left(\begin{array}{c}
z+5 \\
6
\end{array}\right)+\left(\begin{array}{c}
z+1 \\
6
\end{array}\right)\right)+ \\
& \delta_{2}\left(\left(\begin{array}{c}
z+4 \\
6
\end{array}\right)+\left(\begin{array}{c}
z+2 \\
6
\end{array}\right)\right)+\delta_{3}\left(\begin{array}{c}
z+3 \\
6
\end{array}\right) .
\end{aligned}
$$


Substituting $z=-1 / 2+\beta i$ in $L_{P}(z)$ and multiplying by -6 ! gives:

$$
\begin{aligned}
G(\beta):=(2+ & \left.2 \delta_{1}+2 \delta_{2}+\delta_{3}\right) \beta^{6}-\frac{5}{4}\left(202+82 \delta_{1}+10 \delta_{2}-7 \delta_{3}\right) \beta^{4} \\
& +\frac{1}{16}\left(24278+1478 \delta_{1}-682 \delta_{2}+259 \delta_{3}\right) \beta^{2}-\frac{45}{64}\left(462-42 \delta_{1}+14 \delta_{2}-5 \delta_{3}\right) .
\end{aligned}
$$

This is a cubic in $\beta^{2}$, with $G(\beta)=f_{6}\left(\beta^{2}\right)$, and $G(\beta)=0$ if and only if $L_{P}(-1 / 2+\beta i)=0$. The three cases follow from applying Lemma 33 to $f_{6}$. The bounds $\delta_{1} \leqslant 722, \delta_{2} \leqslant 10543$, $\delta_{3} \leqslant 23548$ are clear in cases (i) and (ii); in case (iii) they follow from a positive linear combination of the first three inequalities.

Theorem 35. Let $P$ be a 7 -dimensional reflexive polytope with $\delta$-vector $\left(1, \delta_{1}, \delta_{2}, \delta_{3}, \delta_{3}\right.$, $\left.\delta_{2}, \delta_{1}, 1\right)$. Then $P$ is a CL-polytope if and only if either

(i) $\delta_{1}=2179, \delta_{2}=60657$, and $\delta_{3}=259723$;

(ii) $10882 \delta_{1}=81 \delta_{3}+2674315,10882 \delta_{2}=2477 \delta_{3}+16735603$, and $\delta_{3}<259723$; or

(iii) $139+67 \delta_{1}+19 \delta_{2}>5 \delta_{3}, 8197+1237 \delta_{1}+37 \delta_{3}>203 \delta_{2}, 88069+429 \delta_{2}>3043 \delta_{1}+75 \delta_{3}$, and $\Delta\left(f_{7}\right) \geqslant 0$.

In particular, if $P$ is a CL-polytope then $\delta_{1} \leqslant 2179, \delta_{2} \leqslant 60657$, and $\delta_{3} \leqslant 259723$.

Proof. From Lemma 1, we obtain

$$
\begin{aligned}
L_{P}(z)=\left(\begin{array}{c}
z+7 \\
7
\end{array}\right)+ & \left(\begin{array}{l}
z \\
7
\end{array}\right)+\delta_{1}\left(\left(\begin{array}{c}
z+6 \\
7
\end{array}\right)+\left(\begin{array}{c}
z+1 \\
7
\end{array}\right)\right)+ \\
& \delta_{2}\left(\left(\begin{array}{c}
z+5 \\
7
\end{array}\right)+\left(\begin{array}{c}
z+2 \\
7
\end{array}\right)\right)+\delta_{3}\left(\left(\begin{array}{c}
z+4 \\
7
\end{array}\right)+\left(\begin{array}{c}
z+3 \\
7
\end{array}\right)\right) .
\end{aligned}
$$

Then $L_{P}(z)$ can be divided by $z+1 / 2$ (because $-1 / 2$ is a root). Set

$$
f(z):=-7 ! L_{P}(z) / 2(z+1 / 2) .
$$

The result also follows from Lemma 33 by considering $f(-1 / 2+\beta i)=f_{7}\left(\beta^{2}\right)$. In case (iii) the bounds $\delta_{1} \leqslant 2179, \delta_{2} \leqslant 60657, \delta_{3} \leqslant 259723$ follow from a positive linear combination of the first three inequalities.

Corollary 36. Let $P$ be a 6 - or 7-dimensional $C L$-polytope. Then, $\left|P \cap \mathbb{Z}^{6}\right| \leqslant 3^{6}$ or $\left|P \cap \mathbb{Z}^{7}\right| \leqslant 3^{7}$, respectively.

\subsection{Six- and seven-dimensional real reflexive polytopes}

By making the substitution $z=-1 / 2+\alpha$ in $L_{P}(z)$ we readily obtain the following two theorems:

Theorem 37. Let $P$ be a 6 -dimensional reflexive polytope with $\delta$-vector $\left(1, \delta_{1}, \delta_{2}, \delta_{3}, \delta_{2}\right.$, $\left.\delta_{1}, 1\right)$. Then $P$ is real if and only if one of the following holds: 
(i) $\delta_{1}=722, \delta_{2}=10543$, and $\delta_{3}=23548$;

(ii) $994 \delta_{1}=27 \delta_{3}+81872,497 \delta_{2}=218 \delta_{3}+106407$, and $\delta_{3}>23548$; or

(iii) $202+82 \delta_{1}+10 \delta_{2}>7 \delta_{3}, 24278+1478 \delta_{1}+259 \delta_{3}>682 \delta_{2}, 462+14 \delta_{2}<42 \delta_{1}+5 \delta_{3}$, and $\Delta\left(f_{6}\right) \geqslant 0$.

Theorem 38. Let $P$ be a 7-dimensional reflexive polytope with $\delta$-vector $\left(1, \delta_{1}, \delta_{2}, \delta_{3}, \delta_{3}\right.$, $\left.\delta_{2}, \delta_{1}, 1\right)$. Then $P$ is real if and only if either

(i) $\delta_{1}=2179, \delta_{2}=60657$, and $\delta_{3}=259723$;

(ii) $10882 \delta_{1}=81 \delta_{3}+2674315,10882 \delta_{2}=2477 \delta_{3}+16735603$, and $\delta_{3}>259723$; or

(iii) $139+67 \delta_{1}+19 \delta_{2}<5 \delta_{3}, 8197+1237 \delta_{1}+37 \delta_{3}>203 \delta_{2}, 88069+429 \delta_{2}<3043 \delta_{1}+75 \delta_{3}$, and $\Delta\left(f_{7}\right) \geqslant 0$.

\section{Hypothesis (HS) and an example in dimension ten}

\subsection{Hypothesis (HS)}

In this section we prove Theorem 7 . We actually prove a stronger result:

Theorem 39. Let $P$ be a d-dimensional reflexive polytope and let $\alpha \in \mathbb{C}$ be a root of $L_{P}$.

(i) If $\alpha \in \mathbb{R}$ then $\alpha$ satisfies $-\lfloor d / 2\rfloor<\alpha<\lfloor d / 2\rfloor-1$.

(ii) If $d \leqslant 5$ then $\alpha$ satisfies $-\lfloor d / 2\rfloor<\mathfrak{R e}(\alpha)<\lfloor d / 2\rfloor-1$.

Proof. First we prove case (i). Let $\left(1, \delta_{1}, \ldots, \delta_{\lfloor d / 2\rfloor}, \ldots, \delta_{1}, 1\right)$ be the $\delta$-vector of $P$, where $\delta_{i} \in \mathbb{Z}_{\geqslant 1}$ for each $1 \leqslant i \leqslant\lfloor d / 2\rfloor$. By Lemma 1 we have that $L_{P}(z)=\sum_{i=0}^{d} \delta_{i}\left(\begin{array}{c}n+d-i \\ d\end{array}\right)$. Define

$$
N_{i}(z):=\prod_{j=0}^{d-1}(z+d-i-j)+\prod_{j=0}^{d-1}(z+i-j), \quad \text { for each } 0 \leqslant i \leqslant\lfloor d / 2\rfloor-1,
$$

and

$$
N_{\lfloor d / 2\rfloor}(z):= \begin{cases}\prod_{j=0}^{d-1}\left(z+\frac{d}{2}-j\right), & \text { when } d \text { is even; } \\ \prod_{j=0}^{d-1}\left(z+\frac{d+1}{2}-j\right)+\prod_{j=1}^{d}\left(z+\frac{d-1}{2}-j\right), & \text { when } d \text { is odd }\end{cases}
$$

Then $L_{P}(z)=\sum_{i=0}^{\lfloor d / 2\rfloor} \delta_{i} N_{i}(z) / d$ !. Set

$$
f(z):=d ! L_{P}\left(z-\frac{1}{2}\right)=\sum_{i=0}^{\lfloor d / 2\rfloor} \delta_{i} N_{i}\left(z-\frac{1}{2}\right) .
$$


It is sufficient to prove that all the real roots of $f$ are contained in the open interval $(-\lfloor d / 2\rfloor+1 / 2,\lfloor d / 2\rfloor-1 / 2)$. Note that $f$ satisfies

$$
f(z)=(-1)^{d} f(-z)
$$

For $N_{i}(z-1 / 2), 0 \leqslant i \leqslant\lfloor d / 2\rfloor$, we have the following equalities:

$$
\begin{aligned}
N_{i}\left(z-\frac{1}{2}\right) & =\prod_{j=0}^{d-1}\left(z+d-\frac{1}{2}-i-j\right)+\prod_{j=0}^{d-1}\left(z-\frac{1}{2}+i-j\right) \\
& =\prod_{l=0}^{2 i-1}\left(z-\frac{1}{2}+i-l\right) M_{i}(z),
\end{aligned}
$$

where

$$
M_{i}(z):=\prod_{j=0}^{d-2 i-1}\left(z+\frac{1}{2}+i+j\right)+\prod_{j=0}^{d-2 i-1}\left(z-\frac{1}{2}-i-j\right) .
$$

The coefficients of $M_{i}$ are positive rational numbers when the parity of the degree of $z$ equals the parity of $d$, and its coefficients are 0 when the parity of the degree of $z$ is different from the parity of $d$. Let $\alpha$ be a real number with $\alpha \geqslant\lfloor d / 2\rfloor-1 / 2$. Since $\alpha>0$ we have that $M_{i}(\alpha)>0$. In addition, $\prod_{l=0}^{2 i-1}(\alpha-(1 / 2-i+l))>0$, since $0 \leqslant l \leqslant 2 i-1$ and $0 \leqslant i \leqslant\lfloor d / 2\rfloor$. Hence $\alpha$ cannot be a root of $f$ (since each $\delta_{i}$ is positive). Moreover, by (9), for a real number $\beta$ with $\beta \leqslant-\lfloor d / 2\rfloor+1 / 2, \beta$ cannot be a root of $f(z)$.

We have already shown that case (ii) holds when $d=2$ and $d=3$ (Propositions 9 and 10, respectively). When $d=4$ the roots of $L_{P}(z)$ fall into the four cases (a)-(d) described at the beginning of $\S 3$. Of these, (a)-(c) satisfy (ii): either a root $\alpha$ is of the form $-1 / 2 \pm b i$, and so $\mathfrak{R e}(\alpha)=-1 / 2$, or $\alpha$ is real and so is covered by (i). The only remaining possibility is (d), in which case Proposition 24 gives the result. Similarly, when $d=5$ the result follows from Proposition 31 .

\subsection{Realising a simplex from a $\delta$-vector}

In $\S 6.3$ we give a 10 -dimensional reflexive polytope failing to satisfy (HS). Finding an integer-valued palindromic vector whose numerics give an example is straight-forward; the difficulty lies in showing that this vector is the $\delta$-vector for a lattice polytope. The method we describe below can be used to find all lattice polytopes $P$ with palindromic vector of the form $\left(1,1, \delta_{2}, \ldots, \delta_{\lfloor d / 2\rfloor}, \ldots, \delta_{2}, 1,1\right)$ of length $d+1$.

Let $\delta=\left(1,1, \delta_{2}, \ldots, \delta_{\lfloor d / 2\rfloor}, \ldots, \delta_{2}, 1,1\right)$ be of length $d+1$, where $\delta_{2}, \ldots, \delta_{\lfloor d / 2\rfloor} \in \mathbb{Z}_{\geqslant 1}$, and suppose that this is the $\delta$-vector for a lattice polytope $P$ of dimension $d$. Since $\delta$ is palindromic, $P$ is necessarily reflexive. In particular the origin is the only (strict) interior lattice point of $P$. Since $\delta_{1}=\left|P \cap \mathbb{Z}^{d}\right|-d-1=1$ we know that $P$ is a simplex with $\partial P \cap \mathbb{Z}^{d}=\operatorname{vert}(P)$. Hence $P$ is a terminal reflexive Fano simplex. The corresponding toric variety $X$ given by the spanning fan of $P$ is a Gorenstein fake weighted projective 
space: there exists some weighted projective space $Y=\mathbb{P}\left(\lambda_{0}, \ldots, \lambda_{d}\right)$ with well-formed weights $\left(\lambda_{0}, \ldots, \lambda_{d}\right) \in \mathbb{Z}_{\geqslant 0}^{d}$ such that $X$ is the quotient of $Y$ by the action of a finite group $G$ acting free in codimension one. For details see $[7,8,17]$. By well-formed we mean that $\operatorname{gcd}\left\{\lambda_{0}, \ldots, \widehat{\lambda_{i}}, \ldots, \lambda_{d}\right\}=1$ for each $i \in\{0, \ldots, d\}$, where $\widehat{\lambda_{i}}$ indicates that the $i$-th weight $\lambda_{i}$ is omitted.

Let $N^{\prime} \subset \mathbb{Z}^{d}$ be the sublattice generated by vert $(P)$. Then $G=\mathbb{Z}^{d} / N^{\prime}$ and the order of $G$ is given by the index $\operatorname{mult}(P):=\left[\mathbb{Z}^{d}: N^{\prime}\right]$, which we call the multiplicity of $P$. Restricting $P$ to the sublattice $N^{\prime}$ recovers the simplex $Q$ associated with the weighted projective space $Y[4$, Proposition 2]. Moreover, there exists a matrix $H$ in Hermite normal form with $\operatorname{det}(H)=\operatorname{mult}(P)$ such that

$$
P \cong Q \cdot H
$$

Hence $\operatorname{vol}(P)=\operatorname{mult}(P) \cdot \operatorname{vol}(Q)$. In particular,

$$
\operatorname{mult}(P) \mid d ! \operatorname{vol}(P)=\sum_{i=0}^{d} \delta_{i}
$$

and so the possible multiplicities are determined by $\delta$. The volume $d ! \operatorname{vol}(Q)=h$, where $h:=\lambda_{0}+\cdots+\lambda_{d}$ is defined to be the sum of the weights of $Q$ (and is equal to the Fano index of $Y$ ), so that $h$ is uniquely determined for each choice of multiplicity.

Assume that we have chosen a multiplicity, and consequently have fixed a value for $h$. Since $P$ is terminal and reflexive we have that $Q$ must be terminal and reflexive $[17$, Corollary 2.4 and Corollary 2.5]. By, for example, [9, Lemma 3.5.6], we have that $Q$ is reflexive if and only if

$$
\lambda_{i} \mid h, \quad \text { for each } i \in\{0, \ldots, d\} .
$$

Without loss of generality we may assume that the weights are ordered $\lambda_{0} \leqslant \ldots \leqslant \lambda_{d}$. Since $Q$ is terminal, by [17, Theorem 3.5] we have the strict inequalities

$$
\frac{\lambda_{i}}{h}<\frac{1}{d-i+2}, \quad \text { for each } i \in\{2, \ldots, d\} .
$$

Using (12) and (13) the possible choices of weights for $Q$ can be listed for any given $h$.

Suppose now that we have chosen some possible weights $\left(\lambda_{0}, \ldots, \lambda_{d}\right)$ for $Q$.

(i) The inequalities given by (13) are necessary but not sufficient to guarantee that $Q$ is terminal, however this is easily verified [18, Proposition 3.3]: $Q$ is terminal if and only if

$$
\sum_{i=0}^{d}\left\{\frac{\lambda_{i} \kappa}{h}\right\} \in\{2, \ldots, d-1\}, \quad \text { for each } \kappa \in\{2, \ldots, h-2\}
$$

where $\{a / b\}$ denotes the fractional part of $a / b \in \mathbb{Q}$. 
(ii) Since $P$ is reflexive, we have that $d ! \operatorname{vol}\left(P^{*}\right) \in \mathbb{Z}$. But,

$$
d ! \operatorname{vol}\left(P^{*}\right)=d ! \operatorname{vol}\left(Q^{*}\right) / \operatorname{mult}(P)
$$

and $d ! \operatorname{vol}\left(Q^{*}\right)$ is simply the anticanonical degree of $Y$, given by

$$
d ! \operatorname{vol}\left(Q^{*}\right)=\left(-K_{Y}\right)^{d}=\frac{h^{d}}{\prod_{i=0}^{d} \lambda_{i}} .
$$

Hence we have the requirement that:

$$
\operatorname{mult}(P) \mid \frac{h^{d}}{\prod_{i=0}^{d} \lambda_{i}}
$$

(iii) Since $N^{\prime} \hookrightarrow \mathbb{Z}^{d}$ we require that

$$
L_{Q}(m) \leqslant L_{P}(m), \quad \text { for all } m \in \mathbb{Z}_{\geqslant 0},
$$

where $L_{P}$ is determined by the target $\delta$-vector via Lemma 1 . The $\delta$-vector for $Q$ can be easily computed [18]:

$$
\delta_{j}:=\left|\left\{\kappa \in\{0, \ldots, h-1\} \mid \sum_{i=0}^{d}\left\{\frac{\lambda_{i} \kappa}{h}\right\}=j\right\}\right|, \quad \text { where } j \in\{0, \ldots, d\} .
$$

Hence the Ehrhart polynomial $L_{Q}$ can be computed and the condition verified. Notice that the leading coefficient of $L_{Q}$ is, by construction, at most equal to the leading coefficient of $L_{P}$ (since this are equal to $\operatorname{vol}(Q)$ and $\operatorname{vol}(P)$ respectively), hence (14) is automatically satisfied for all sufficiently large values of $m$.

In practice, these three conditions are sufficiently strong as to exclude many candidate weights, and this is often sufficient to show that no such $Q$ exists, or to restrict the possibilities to only one or two cases.

Finally, for each choice of weights $\left(\lambda_{0}, \ldots, \lambda_{d}\right)$ satisfying the above conditions, one can simply work through the possible Hermite normal forms $H$ with $\operatorname{det}(H)=\operatorname{mult}(P)$ and consider the resulting simplicies arising from (10). Here one can exploit the symmetries of $Q$ arising from the weights, and the fact that $Q$ is reflexive, in order to reduce the number of choices of $H$ that need to be considered.

\subsection{An example in dimension ten}

Let $\delta=(1,1,1,1,9,28,9,1,1,1,1)$. It is easily verified that the corresponding Ehrhart polynomial has a root $\alpha$ with $\mathfrak{R e}(\alpha)<-5$ and a $\operatorname{root} \beta$ with $\mathfrak{R e}(\beta)>4$. We shall use the method sketched above to construct a simplex $P$ with this $\delta$-vector. We will make considerable use of the combinatorics of cyclic quotient singularities; an excellent reference is [24]. 


\begin{tabular}{|c|c|}
\hline $\operatorname{mult}(P)$ & $\left(\lambda_{0}, \ldots, \lambda_{10}\right)$ \\
\hline 1 & $(1,1,1,1,1,1,6,6,9,9,18)$ \\
\hline 1 & $(1,1,1,1,2,3,3,6,9,9,18)$ \\
\hline 1 & $(1,1,1,1,2,3,6,6,6,9,18)$ \\
\hline 1 & $(1,1,1,1,2,6,6,9,9,9,9)$ \\
\hline 1 & $(1,1,1,2,2,2,3,6,9,9,18)$ \\
\hline 1 & $(1,1,1,2,2,2,6,6,6,9,18)$ \\
\hline 1 & $(1,1,2,2,2,2,2,6,9,9,18)$ \\
\hline 1 & $(1,1,2,2,3,3,3,3,9,9,18)$ \\
\hline 1 & $(1,1,2,2,3,3,3,6,6,9,18)$ \\
\hline 1 & $(1,1,2,2,3,3,6,6,6,6,18)$ \\
\hline 1 & $(1,1,2,2,3,3,6,9,9,9,9)$ \\
\hline 1 & $(1,1,2,2,3,6,6,6,9,9,9)$ \\
\hline
\end{tabular}

\begin{tabular}{|c|c|}
\hline $\operatorname{mult}(P)$ & $\left(\lambda_{0}, \ldots, \lambda_{10}\right)$ \\
\hline 1 & $(1,1,2,2,6,6,6,6,6,9,9)$ \\
\hline 1 & $(1,2,2,2,2,3,3,3,9,9,18)$ \\
\hline 1 & $(1,2,2,2,2,3,3,6,6,9,18)$ \\
\hline 1 & $(1,2,2,2,2,3,6,9,9,9,9)$ \\
\hline 1 & $(1,2,2,2,2,6,6,6,9,9,9)$ \\
\hline 1 & $(2,2,2,2,2,2,3,3,9,9,18)$ \\
\hline 3 & $(1,1,1,1,1,1,1,1,1,3,6)$ \\
\hline 3 & $(1,1,1,1,1,1,1,1,2,2,6)$ \\
\hline 3 & $(1,1,1,1,1,1,1,2,3,3,3)$ \\
\hline 3 & $(1,1,1,1,1,1,2,2,2,3,3)$ \\
\hline 3 & $(1,1,1,1,1,2,2,2,2,2,3)$ \\
\hline 3 & $(1,1,1,1,2,2,2,2,2,2,2)$ \\
\hline
\end{tabular}

Table 2: The initial list of candidate Gorenstein terminal weights from which we may be able to construct a terminal reflexive simplex with $\delta$-vector $(1,1,1,1,9,28,9,1,1,1,1)$.

By (11) we have that $\operatorname{mult}(P) \in\{1,2,3,6,9,18,27,54\}$. Using (12) and (13) and checking conditions (i) and (ii) we obtain a list of 24 candidate well-formed Gorenstein terminal weights; see Table 2 . Of these, all but $(1,1,1,1,1,1,2,2,2,3,3)=\left(1^{6}, 2^{3}, 3^{2}\right)$ with mult $(P)=3$ are excluded by condition (iii).

We have reduced to the case where $X=\mathbb{P}\left(1^{6}, 2^{3}, 3^{2}\right) / G,|G|=3$. Hence $G$ is a cyclic group. Write $\frac{1}{3}\left(\alpha_{0}, \ldots, \alpha_{10}\right)$ for a generator of $G$, where $\alpha_{i} \in\{0,1,2\}$. This acts on $Y=\mathbb{P}\left(1^{6}, 2^{3}, 3^{2}\right)$ via

$$
\left(x_{0}, \ldots, x_{10}\right) \mapsto\left(\varepsilon^{\alpha_{0}} x_{0}, \ldots, \varepsilon^{\alpha_{10}} x_{10}\right), \quad \text { where } \varepsilon^{3}=1 .
$$

The fixed-points of this group-action are unchanged when we include the action of the weights; that is, the transformation

$$
\frac{1}{3}\left(\alpha_{0}, \ldots, \alpha_{10}\right) \mapsto \frac{1}{3}\left(\overline{\alpha_{0}+\lambda_{0}}, \ldots, \overline{\alpha_{10}+\lambda_{10}}\right)
$$

leaves $X$ unchanged. Here $\bar{a}$ denotes the unique integer $0 \leqslant c<3$ such that $a \equiv c \bmod 3$. Since $\lambda_{0}=1$ we use this transformation to arrange for $\alpha_{0}=0$.

That $\lambda_{0}=1$ means that the affine chart on $Y$ given by setting $x_{0}=1$ is smooth. We can interpret this combinatorially as follows. Let $v_{0}, \ldots, v_{10} \in N^{\prime}$ be the vertices of $Q$, satisfying $\lambda_{0} v_{0}+\ldots+\lambda_{10} v_{10}=0$. Restricting to $x_{0}=1$ corresponds to taking the cone over the facet $F$ of $Q$ that does not contain $v_{0}$. That is, $C_{0}:=\operatorname{cone}\left\{v_{1}, \ldots, v_{10}\right\}$. That $C_{0}$ is smooth means that the generators $v_{1}, \ldots, v_{10}$ form a basis for the lattice $N^{\prime}$. The action of the group $G$ on this chart is given by $\frac{1}{3}\left(\alpha_{1}, \ldots, \alpha_{10}\right)$; we regard this as a rational point $g=\frac{1}{3} \sum_{i=1}^{10} \alpha_{i} v_{i}$ in $N^{\prime} \otimes_{\mathbb{Z}} \mathbb{Q}$. Let $N^{\prime} \hookrightarrow N^{\prime}+g \cdot \mathbb{Z}$ be the natural inclusion of the lattice $N^{\prime}$ in the lattice $N^{\prime}+g \cdot \mathbb{Z}$ generated by adding $g$. We identify $C_{0}$ with its image 
under this embedding. By assumption $C_{0}$ is a Gorenstein cone. But $C_{0}$ is Gorenstein if and only if

$$
3 \mid \sum_{i=1}^{10} \lambda_{i} .
$$

The Ehrhart polynomials of $P$ and $Q$ are readily calculated from their respective $\delta$-vectors:

$$
\begin{aligned}
& L_{P}(m)=\frac{18}{10 !}\left(3 m^{10}+15 m^{9}+225 m^{8}+810 m^{7}+17969 m^{6}+51135 m^{5}+\right. \\
& \left.274775 m^{4}+465240 m^{3}+815828 m^{2}+591600 m+201600\right) \\
& \text { and } L_{Q}(m)=\frac{18}{10 !}\left(m^{10}+5 m^{9}+255 m^{8}+990 m^{7}+17843 m^{6}+50085 m^{5}+\right. \\
& \left.274945 m^{4}+467560 m^{3}+815756 m^{2}+590160 m+201600\right)
\end{aligned}
$$

Notice that $L_{P}(m)=L_{Q}(m)$ when $m \in\{0,1,2,3\}$. Let $u \in \operatorname{Hom}\left(N^{\prime}, \mathbb{Z}\right)$ correspond to the height 1 supporting hyperplane of the facet $F$. Then $\left|\left\{v \in C_{0} \cap N^{\prime} \mid u(v) \leqslant 3\right\}\right|=$ $\left|\left\{v \in C_{0} \cap N^{\prime}+g \cdot \mathbb{Z} \mid u(v) \leqslant 3\right\}\right|$. Hence we conclude that

$$
\frac{1}{3} \sum_{i=1}^{10} \overline{\kappa \alpha_{i}} \notin\{1,2,3\}, \quad \text { for } \kappa \in\{1,2\} .
$$

For any permutation $\sigma$ of the integers $\{1, \ldots, 10\}$ such that $\lambda_{\sigma i}=\lambda_{i}$ for each $i \in$ $\{1, \ldots, 10\}$, we can regard $\frac{1}{3}\left(0, \alpha_{1}, \ldots, \alpha_{10}\right)$ and $\frac{1}{3}\left(0, \alpha_{\sigma 1}, \ldots, \alpha_{\sigma 10}\right)$ as generating equivalent group actions. Thus there are 21 choices (up to permutation) for $\alpha_{1}, \ldots, \alpha_{5}$, and 10 choices for $\alpha_{6}, \alpha_{7}, \alpha_{8}$. For any particular choice of $\alpha_{1}, \ldots, \alpha_{8}$, condition (15) means that there are only two choices for $\alpha_{9}$ and $\alpha_{10}$. Furthermore, whether we pick $\frac{1}{3}\left(0, \alpha_{1}, \ldots, \alpha_{10}\right)$ or $\frac{1}{3}\left(0, \overline{2 \alpha_{1}}, \ldots, \overline{2 \alpha_{10}}\right)$ as our generator makes no difference: up to permutation this involution fixes exactly $3 \cdot 2 \cdot 2=12$ cases (including the trivial action). Remembering to exclude the trivial action, a simple counting argument gives us $(21 \cdot 10 \cdot 2-12) / 2+11=215$ distinct group actions.

Condition (16) reduces the 215 possible group actions to just 58 candidates; we call the set of such candidates $\mathcal{G}$. Remembering that our decision to use the weights $\left(1^{6}, 2^{3}, 3^{2}\right)$ to zero $\alpha_{0}$ was a choice, and that we could just have validly chosen to set $\alpha_{i}=0$ for any $i \in\{0, \ldots, 5\}$ (i.e. any $i$ such that $\lambda_{i}=1$ ), we have that for any $\left\langle\frac{1}{3}\left(\alpha_{0}, \ldots, \alpha_{10}\right)\right\rangle \in \mathcal{G}$ we need $\left\langle\frac{1}{3}\left(\overline{\alpha_{0}-\alpha_{i} \lambda_{0}}, \ldots, \overline{\alpha_{10}-\alpha_{i} \lambda_{10}}\right)\right\rangle \in \mathcal{G}$, for each $i \in\{0, \ldots, 5\}$, where now we regard groups as being defined only up to permutations $\sigma$ of $\{0, \ldots, 10\}$ that fix $\lambda_{\sigma i}=\lambda_{i}$. This gives us exactly one possible group action, generated by

$$
\frac{1}{3}(0,1,2,0,1,2,0,1,2,1,2) .
$$

Finally, we compute the resulting polytope $P$ and check that the $\delta$-vector agrees. Up to isomorphism,

$$
P=\operatorname{conv}\left\{e_{1}, \ldots, e_{9},(1,2,0,1,2,0,1,1,2,3),(-4,-5,-2,-3,-4,-1,-2,-2,-3,-3)\right\},
$$

where $e_{i}$ is the $i$-th standard basis element, and $P$ has $\delta$-vector $(1,1,1,1,9,28,9,1,1,1,1)$. 


\section{References}

[1] Victor V. Batyrev. Dual polyhedra and mirror symmetry for Calabi-Yau hypersurfaces in toric varieties. J. Algebraic Geom., 3(3):493-535, 1994.

[2] M. Beck, J. A. De Loera, M. Develin, J. Pfeifle, and R. P. Stanley. Coefficients and roots of Ehrhart polynomials. In Integer points in polyhedra-geometry, number theory, algebra, optimization, volume 374 of Contemp. Math., pages 15-36. Amer. Math. Soc., Providence, RI, 2005.

[3] Christian Bey, Martin Henk, and Jörg M. Wills. Notes on the roots of Ehrhart polynomials. Discrete Comput. Geom., 38(1):81-98, 2007.

[4] A. A. Borisov and L. A. Borisov. Singular toric Fano three-folds. Mat. Sb., 183(2):134-141, 1992. text in Russian. English transl.: Russian Acad. Sci. Sb. Math., 75 (1993), 277-283.

[5] Benjamin Braun. Norm bounds for Ehrhart polynomial roots. Discrete Comput. Geom., 39(1-3):191-193, 2008.

[6] Benjamin Braun and Mike Develin. Ehrhart polynomial roots and Stanley's nonnegativity theorem. In Integer points in polyhedra-geometry, number theory, representation theory, algebra, optimization, statistics, volume 452 of Contemp. Math., pages 67-78. Amer. Math. Soc., Providence, RI, 2008.

[7] Weronika Buczyńska. Fake weighted projective spaces. arXiv:0805.1211v1, May 2008.

[8] Heinke Conrads. Weighted projective spaces and reflexive simplices. Manuscripta Math., 107(2):215-227, 2002.

[9] David A. Cox and Sheldon Katz. Mirror symmetry and algebraic geometry, volume 68 of Mathematical Surveys and Monographs. American Mathematical Society, Providence, RI, 1999.

[10] Eugène Ehrhart. Sur les polyèdres homothétiques bordés à $n$ dimensions. $C . R$. Acad. Sci. Paris, 254:988-990, 1962.

[11] Eugène Ehrhart. Sur un problème de géométrie diophantienne linéaire. II. Systèmes diophantiens linéaires. J. Reine Angew. Math., 227:25-49, 1967.

[12] V. V. Golyshev. On the canonical strip. Uspekhi Mat. Nauk, 64(1(385)):139-140, 2009.

[13] Gábor Hegedüs and Alexander M. Kasprzyk. Roots of Ehrhart polynomials of smooth Fano polytopes. Discrete Comput. Geom., 46(3):488-499, 2011.

[14] Takayuki Hibi. Ehrhart polynomials of convex polytopes, $h$-vectors of simplicial complexes, and nonsingular projective toric varieties. In Discrete and computational geometry (New Brunswick, NJ, 1989/1990), volume 6 of DIMACS Ser. Discrete Math. Theoret. Comput. Sci., pages 165-177. Amer. Math. Soc., Providence, RI, 1991. 
[15] Takayuki Hibi. A lower bound theorem for Ehrhart polynomials of convex polytopes. Adv. Math., 105(2):162-165, 1994.

[16] Akihiro Higashitani. Counterexamples of the conjecture on roots of Ehrhart polynomials. Discrete Comput. Geom., 47(3):618-623, 2012.

[17] Alexander M. Kasprzyk. Bounds on fake weighted projective space. Kodai Math. J., 32:197-208, 2009.

[18] Alexander M. Kasprzyk. Classifying terminal weighted projective space. arXiv:1304.3029 [math.AG], 2013.

[19] Alexander M. Kasprzyk and Benjamin Nill. Fano polytopes. In Anton Rebhan, Ludmil Katzarkov, Johanna Knapp, Radoslav Rashkov, and Emanuel Scheidegger, editors, Strings, Gauge Fields, and the Geometry Behind - the Legacy of Maximilian Kreuzer, pages 349-364. World Scientific, 2012.

[20] Maximilian Kreuzer and Harald Skarke. Complete classification of reflexive polyhedra in four dimensions. Adv. Theor. Math. Phys., 4(6):1209-1230, 2000.

[21] I. G. Macdonald. Polynomials associated with finite cell-complexes. J. London Math. Soc. (2), 4:181-192, 1971.

[22] Tetsushi Matsui, Akihiro Higashitani, Yuuki Nagazawa, Hidefumi Ohsugi, and Takayuki Hibi. Roots of Ehrhart polynomials arising from graphs. J. Algebraic Combin., 34(4):721-749, 2011.

[23] Hidefumi Ohsugi and Kazuki Shibata. Smooth Fano polytopes whose Ehrhart polynomial has a root with large real part. Discrete Comput. Geom., 47(3):624-628, 2012.

[24] Miles Reid. Young person's guide to canonical singularities. In Algebraic geometry, Bowdoin, 1985 (Brunswick, Maine, 1985), volume 46 of Proc. Sympos. Pure Math., pages 345-414. Amer. Math. Soc., Providence, RI, 1987.

[25] Fernando Rodriguez-Villegas. On the zeros of certain polynomials. Proc. Amer. Math. Soc., 130(8):2251-2254 (electronic), 2002.

[26] Richard P. Stanley. Decompositions of rational convex polytopes. Ann. Discrete Math., 6:333-342, 1980. Combinatorial mathematics, optimal designs and their applications (Proc. Sympos. Combin. Math. and Optimal Design, Colorado State Univ., Fort Collins, Colo., 1978). 